\title{
A Transcriptional Program Promotes Remodeling of GABAergic Synapses in Caenorhabditis elegans
}

\author{
Sarah C. Petersen, ${ }^{1}$ Joseph D. Watson, ${ }^{1}$ Janet E. Richmond, ${ }^{2}$ Mihail Sarov, ${ }^{3}$ Walter W. Walthall, ${ }^{4}$ \\ and David M. Miller III ${ }^{1,5}$ \\ ${ }^{1}$ Department of Cell and Developmental Biology, Vanderbilt University Medical Center, Nashville, Tennessee 37232, ${ }^{2}$ Department of Biological Sciences, \\ University of Illinois at Chicago, Chicago, Illinois 60607, ${ }^{3}$ TransgeneOmics Facility, Max Planck Institute of Molecular Cell Biology and Genetics, D-01307 \\ Dresden, Germany, ${ }^{4}$ Department of Biology, Georgia State University, Atlanta, Georgia 30302, and ${ }^{5}$ Vanderbilt Kennedy Center and Program in \\ Neuroscience, Nashville, Tennessee 37203
}

Although transcription factors are known to regulate synaptic plasticity, downstream genes that contribute to neural circuit remodeling are largely undefined. In Caenorhabditis elegans, GABAergic Dorsal D (DD) motor neuron synapses are relocated to new sites during larval development. This remodeling program is blocked in Ventral D (VD) GABAergic motor neurons by the COUP-TF (chicken ovalbumin upstream promoter transcription factor) homolog, UNC-55. We exploited this UNC-55 function to identify downstream synaptic remodeling genes that encode a diverse array of protein types including ion channels, cytoskeletal components, and transcription factors. We show that one of these targets, the Iroquois-like homeodomain protein, IRX-1, functions as a key regulator of remodeling in DD neurons. Our discovery of irx-1 as an unc-55-regulated target defines a transcriptional pathway that orchestrates an intricate synaptic remodeling program. Moreover, the well established roles of these conserved transcription factors in mammalian neural development suggest that a similar cascade may also control synaptic plasticity in more complex nervous systems.

\section{Introduction}

Synaptic networks are remodeled by developmental signals and experience (Barnes and Finnerty, 2010). This plasticity is embodied by structural changes that include the pruning or extension of neurites and the relocation of synapses (Ding et al., 2007; Hashimoto et al., 2009). Both developmental and activitydependent mechanisms of synaptic plasticity can be controlled by transcription. For example, Drosophila mushroom body neuron remodeling is regulated by the antagonistic functions of orphan nuclear hormone receptors (Boulanger et al., 2011). In mammals, the activity-dependent role of myocyte enhancer factor 2 (MEF2) transcription factor limits synaptogenesis in excitatory

Received June 22, 2011; revised Aug. 16, 2011; accepted Sept. 6, 2011.

Author contributions: S.C.P., J.D.W., J.E.R., and D.M.M. designed research; S.C.P., J.D.W., and J.E.R. performed research; M.S. and W.W.W. contributed unpublished reagents/analytic tools; S.C.P., J.D.W., J.E.R., and D.M.M. analyzed data; S.C.P. and D.M.M. wrote the paper.

This work was supported by National Institutes of Health (NIH) Grants R21 MH077302 (D.M.M.), R01 NS26115 (D.M.M.), T32 GM08554 (S.C.P.), F31 NS063669 (S.C.P.), F31 NS049743 (J.D.W.), and R01 MH073156 (J.E.R.). Some of the strains used for this study were provided by the Caenorhabditis Genetics Center, which is funded by the NIH National Center for Research Resources, and by the Japanese National BioResource Project. We thank M. Nonet for providing juls1, B. Bamber for oxls22, M. Zhen for providing hpls3, S. Levy and J. Mote in the Vanderbilt Genome Sciences Resource for assistance with gene expression profiling, K. Watkins for assistance with creating wdls31, S. Von Stetina and W. C. Spencer for help with microarray experiments, and members of the Miller Laboratory for advice.

The authors declare no competing financial interests.

Correspondence should be addressed to David M. Miller III, Department of Cell and Developmental Biology, Vanderbilt University Medical Center, 3120 MRBIII, 465 21st Avenue South, Nashville, TN 37232. E-mail: david.miller@vanderbilt.edu.

J. D. Watson's present address: Postdoctoral Fellow, Department of Biochemistry and Biophysics, University of North Carolina, Chapel Hill, Chapel Hill, NC 27599.

DOI:10.1523/JNEUROSCI.3181-11.2011

Copyright $\odot 2011$ the authors $\quad 0270-6474 / 11 / 3115362-14 \$ 15.00 / 0$ hippocampal circuits. The precise orchestration of these complex events by transcription factors suggests that identification of downstream targets could reveal important molecular components of synaptic remodeling programs (Flavell et al., 2008). Here, we describe a strategy that exploits this approach to discover new determinants of developmentally regulated synaptic plasticity in Caenorhabditis elegans.

The C. elegans Dorsal D (DD) GABAergic motor neurons display stereotypical changes in synaptic location during early larval development. In this developmental program, initially ventral DD synapses are relocated to new positions on the dorsal side with no evident alterations in DD process morphology (White et al., 1978). The timing of this event depends in part on the heterochronic gene lin-14, but the mechanism of its role is unknown (Hallam and Jin, 1998). A recent study has shown that DD synaptic components from the ventral side are reassembled in dorsal synapses through the action of CYY-1 cyclin box-containing protein, which removes ventral presynaptic proteins, and CDK-5 cyclin-dependent kinase, which facilitates trafficking to nascent dorsal synapses (Park et al., 2011).

A second functionally and structurally related class of GABAergic motor neurons, the Ventral Ds (VDs), normally do not remodel due to the cell-autonomous function of the chicken ovalbumin upstream promoter transcription factor (COUP-TF) UNC-55 (Walthall and Plunkett, 1995; Zhou and Walthall, 1998). VD synapses relocate to the dorsal side in unc-55 mutants, and conversely, ectopic expression of UNC-55 protein in DDs blocks dorsal synaptogenesis and forces retention of ventral synapses (Shan et al., 2005). These results indicate that GABAergic motor neuron remodeling depends on transcriptional control of 
downstream genes and that these effector molecules could be revealed by identifying UNC-55 targets. We used a combination of cell-specific profiling and RNAi to identify 19 conserved remodeling genes that encode a diverse spectrum of protein types. We show that one of these $u n c-55$ targets, the Iroquois homeodomain protein, IRX-1, is both necessary and sufficient for GABAergic motor neuron remodeling and thus that IRX-1 functions as a key downstream regulator of synaptic remodeling genes. Although homologs of UNC-55 and of IRX-1 specify cell fate in overlapping domains of developing vertebrate nervous systems (Matsumoto et al., 2004; Cheng et al., 2005; Kim et al., 2009; Tang et al., 2010), our work provides the first indication that they function together in a common pathway to regulate synaptic remodeling. In addition, the suite of downstream genes that we have uncovered provides a rich resource for future studies of the mechanism of synaptic plasticity.

\section{Materials and Methods}

\section{Strains and genetics}

Nematode strains were maintained at $20-25^{\circ} \mathrm{C}$ with standard culture techniques (Brenner, 1974). The wild-type strain is N2 and all references to unc-55 mutants used the allele unc-55(e1170) (Walthall, 1990). This strain contains an insertion (CCCC to CCCCC) in the DNA binding domain and upstream of the ligand binding domain. This frameshift also results in premature termination of the protein at amino acid 26; therefore, the $e 1170$ allele is presumed to be a genetic null. All animals used in this study were hermaphrodites.

\section{Strain list}

Note: unc-119(+) refers to unc-119-rescuing minigene from MM051 (Maduro and Pilgrim, 1995) (see details of strain construction).

General synaptic remodeling analysis strains. CZ333, juIs1[punc-25:: SNB-1::GFP; lin-15+]; NC2296, unc-119; juIs1[punc-25::SNB-1::GFP; lin-15+]; wdEx658[punc-25::mCherry::unc-54UTR, unc-119(+)]; CB1170, unc-55(e1170), >10× outcrossed; NC1851, unc-55(e1170); juIs1 [punc-25:: SNB-1::GFP; lin-15+]; NC2319, unc-55(e1170); unc-119; juIs1 [punc-25::SNB-1::GFP; lin-15+]; wdEx658[punc-25::mCherry::unc-54UTR, unc-119(+)]; EG1653, oxIs22 [punc-49::UNC-49::GFP; lin-15(+)]; NC2122, unc-55; oxIs22 [punc-49::UNC-49::GFP; lin-15(+)]; ZM54, hpIs3[punc-25::SYD-2::GFP; lin-15(+)]; NC1849, unc-55(e1170); hpIs3[punc-25::SYD-2::GFP; lin-15(+)].

mRNA tagging strains. NC1412, dpy-5(e907); wdEx562[pttr-39::3xFLAG:: $P A B-1 d p y-5(+)$ ] (before integration); NC1645, $d p y-5$ (e907); wdIs31 [pttr39::3xFLAG::PAB-1 dpy-5(+)] IV; NC1546, dpy-5(e907) unc-55(e1170); wdIs31[pttr-39::3xFLAG::PAB-1 dpy-5(+)] IV.

RNAi screen strain. NC1852, unc-55(e1170); eri-1(mg366) juIs1 [punc25::SNB-1::GFP; lin-15+].

irx-1::GFP strains. irx-1::GFP strains were as follows: NC2233, unc119(ed3); wdIs78[irx-1::GFP; unc-119+ fosmid]; NC2298, unc-55; unc119(ed3); wdIs78[irx-1::GFP; unc-119+ fosmid].

irx-1(csRNAi) strains. NC2111, unc-119(ed3); wdEx686[pttr-39::irx1::unc-10UTR; unc-119+; punc-25:: mCherry::unc-54UTR; pttr-39::irx-1 antisense::unc-10UTR]; NC2297, unc-119(ed3); juIs1 [punc-25::SNB-1::GFP; lin-15+]; wdEx686[pttr-39::irx-1:: unc-10UTR; unc-119+; punc-25::mCherry:: unc-54UTR; pttr-39::irx-1 antisense::unc-10UTR]; NC2169, unc55(e1170); unc-119(ed3); juIs1 [punc-25::SNB-1::GFP; lin-15+]; wdEx686[pttr-39::irx-1::unc-10UTR; unc-119+; punc-25::mCherry::unc54UTR; pttr-39::irx-1 antisense::unc-10UTR].

Electrophysiology strains. EG5025, oxIs351[punc-47:ChR2::mCherry:: unc-54UTR lin-15+ LITMUS 38i] X; NC2211, unc-55 (e1170); oxIs351[punc-47:ChR2::mCherry::unc- 54UTR lin-15+ LITMUS 38i] X; NC2212, unc-55 (e1170); unc-119(ed3); oxIs351[punc-47:ChR2:: mCherry::unc-54UTR lin-15+ LITMUS 38i] X; wdEx686[pttr-39:: irx-1::unc-10UTR; unc-119+; punc-25::mCherry:: unc-54UTR; pttr-39:: irx-1 antisense::unc-10UTR].

irx-1(gf) strain. NC2347, unc-119(ed3); juIs1[punc-25::SNB-1::GFP; lin-15+]; wdEx753[pttr-39::IRX-1::GFP; unc-119+].
Microscopy and synapse scoring

SNB-1::GFP assays were performed with the transgene juIs1 [punc-25:: SNB-1::GFP; lin-15+] (Hallam and Jin, 1998), SYD-2::GFP assays used hpIs3[punc-25::SYD-2::GFP; lin-15+] (Yeh et al., 2005), and UNC-49::GFP assays used oxIs22 [punc-49::UNC-49::GFP; lin-15+] (Bamber et al., 1999). Animals were anesthetized with $0.1 \%$ tricaine/tetramisole, mounted on a $2 \%$ agarose pad, and imaged with a Zeiss Axiovert microscope using MetaMorph software. Images in Figures 1, 3, 4, 6, and 9 were obtained with a Leica TCSSP5 confocal microscope with Leica Application Suite Advanced Fluorescence (LAS-AF) software and created using maximumintensity projections of $Z$-stacks collected at $1 \mu \mathrm{m} / \mathrm{step}$ with a $40 \times$ objective. Intensity line scans in Figure 3 were created using Image software of confocal $Z$-stack maximum-intensity projections.

For irx-1(csRNAi) quantification (see Fig. 6), individual VD motor neurons expressing mCherry [control or irx-1(csRNAi) labeling] were scored. For each mCherry+ VD, the number of SNB-1::GFP puncta in the anterior interval between the VD and the next anterior VD were counted in the dorsal and ventral nerve cords. These analyses were considered puncta per "VD anterior region."

For irx-1(gf) studies, the $w d E x 753$ transgene was crossed into the juIs1 [punc-25::SNB-1::GFP] background. In this strain, SNB-1::GFP is normally excluded from the nucleus. We performed mosaic analysis by identifying individual VD motor neurons scoring as "wild type" (SNB-1::GFP in cell soma, but no irx-1::GFP in nucleus) or "irx-1 (gf)" (IRX-1::GFP localized in nucleus, with SNB-1::GFP in soma). We observed that very high IRX-1::GFP expression sometimes silenced SNB-1::GFP expression. Therefore, the fluorescence in the nucleus (IRX-1::GFP) and soma (SNB-1::GFP) of every neuron scored was quantified using confocal microscopy and $Z$-stack maximum intensity projections in LAS-AF software. Only neurons with equivalent fluorescent intensity in the nucleus and soma were scored as "irx-1(gf)" neurons. Adjacent neurons with high cell soma intensity/low nuclear intensity were scored as wild-type neurons. For each VD, the number of SNB-1::GFP puncta in the anterior interval between the VD and the next anterior VD were counted in the dorsal and ventral nerve cords. These analyses were considered puncta per VD anterior region.

\section{GABA mRNA-tagging strain}

Gene expression profiling experiments used the transgene wdIs31 [pttr-39::3xFLAG::PAB-1] (Spencer et al., 2011) in both wild-type and unc-55 backgrounds. For unc-55 profiles, a $d p y-5$ (e907) unc-55(e1170) recombinant was generated and crossed into NC1645 to yield NC1546 $d p y-5(e 907)$ unc-55(e1170); wdIs31. wdIs31 transgenics were immunostained with monoclonal mouse anti-FLAG antibodies (Sigma-Aldrich) and goat anti-mouse IgG Cy3 to detect expression of FLAG-PAB-1 (Finney and Ruvkun, 1990). To identify GABA motor neurons, animals were costained with DAPI, mounted on a $2 \%$ agarose pad, and imaged on a Zeiss Axioplan compound microscope. DD and VD motor neurons were identified by their stereotyped locations in the ventral nerve cord.

Detection of unc-55-regulated transcripts from GABA neuron-specific microarray profiling results mRNA tagging methods were as described (Von Stetina et al., 2007b). Briefly, anti-FLAG beads were used to isolate GABA-neuron-specific RNA from lysates of wild-type and unc-55 mutant mid-L2 stage larvae expressing the $w d I s 31$ transgene. Approximately $10 \mathrm{ng}$ of RNA for each sample was amplified with WT-Ovation Pico RNA Amplification System (NuGEN Technologies) and $\sim 5 \mu$ g of labeled target cDNA was hybridized to Affymetrix C. elegans GeneChip arrays. Independent data sets were collected from three wild-type (N2) and four unc-55 mutant mRNA tagging samples. Probe intensities were normalized with robust multiarray analysis. Transcripts showing $\geq 2$-fold difference at $<1 \%$ false discovery rate between wild-type versus unc-55 mutant data sets were identified with two-class unpaired analysis in significance analysis of microarray (SAM) (Irizarry et al., 2003).

\section{Annotation of expression profiles}

Genes were classified based on gene ontology with Perl scripts and hand annotation as previously described (Von Stetina et al., 2007b). Gene ontology was refined using information consolidated at wormbase.org, 
release WS221 (i.e., INTERPRO protein domains), GO terms inferred from mutant phenotype, and gene descriptions contributed to Wormbase with references. Conservation was based on BLASTP E values $\left(<\mathrm{e}^{-10}\right.$ to Homo sapiens) (wormbase.org).

\section{RNAi suppression screen}

The RNAi screen was performed with unc-55(e1170); juIs1; eri-1 (mg366) (Kennedy et al., 2004). The RNAi hypersensitive eri-1 mutation was confirmed by PCR assay (Earls et al., 2010). RNAi knockdown by feeding used bacterial clones from the C. elegans RNAi library (Kamath et al., 2003) with plates created as previously described (Earls et al., 2010) with the exception that no EGTA was added to media. Three L4 hermaphrodites (NC1852) were grown on each RNAi plate at $20-23^{\circ} \mathrm{C}$ for $5 \mathrm{~d}$ until $\mathrm{F}_{2}$ progeny reached young adult stage. SNB-1::GFP puncta in the ventral nerve cord were counted for five or more animals per clone. Negative control RNAi (L4440 containing no genomic insert) was analyzed with each set of clones screened; $i r x-1$ and $u n c-8$ were used as positive controls. Counting was performed with the observer blinded to the identify RNAi clones until after data collection was completed. "Hits" were defined as RNAi clones that resulted in a significant increase in ventral SNB-1::GFP puncta according these criteria: (1) ventral puncta were significantly higher in animals treated with RNAi for a candidate unc-55 target than empty vector ( $p<0.01$, Student's $t$ test); (2) this phenotype was detected in at least one-half of the animals scored (i.e., a penetrance of $>50 \%$ ). For this criterion, "phenotype" means a number of puncta above the empty vector control upper bound ( $\sim 40$ puncta). All conserved hits were rescreened independently to confirm unc-55 suppression. For all hits, the RNAi feeding plasmid was purified from library clones via QIAprep Spin Miniprep kit (QIAGEN) and sequenced to verify the gene identity.

\section{Construction of irx-1::GFP}

GFP and the unc-119 minigene were inserted into a fosmid containing the irx-1 locus (Sarov et al., 2006; Zhong et al., 2010). In this construct, GFP is fused within the irx-1 C terminus and produces a functional protein capable of rescuing the lethality observed in irx-1-null mutants (data not shown). The ir $x-1:: G F P$ fosmid was purified using a modified columnless QIAGEN miniprep protocol (Warming et al., 2005) and unc119 worms were transformed via microparticle bombardment with $8 \mu \mathrm{g}$ of irx-1::GFP fosmid coated on gold beads at 1800 psi as previously described (Spencer et al., 2011). Bombardment yielded a spontaneous integrant, $w d I s 78$.

\section{Movement assays}

For wild-type versus Unc-55 movement, juIs1 (wild type) and unc-55; juIs1 ("unc-55") animals were first tapped on the tail to ensure that they were capable of forward locomotion. Worms were then tapped on the head to assess ability to execute backward locomotion. Animals were binned into the following categories: "severe Unc" (coil ventrally immediately upon tapping), "moderate Unc" (coil ventrally while attempting backward locomotion), and wild type (sustain backward locomotion with at least two body bends). To present the data in Figure 1, all "severe" and "moderate" Unc worms were grouped into a single "uncoordinated" category. For irx-1 global (i.e., feeding) RNAi movement assays, unc-55; juIs1 L4 larvae were treated with empty vector and irx-1 RNAi as described above with the exception that eri-1(mg366) was not used in this assay. In this case, worms were tapped on the head to assess suppression of the Unc-55 backward movement defect and were binned into severe Unc, moderate Unc, and wild-type categories as described above.

\section{Construction of irx-1(csRNAi)}

Whole-worm cDNA was generated by RT-PCR of mixed-stage N2 RNA with poly-dT (Affy 100) primers and SuperScript II (Invitrogen). Two microliters of the RT-PCR was used as template to PCR amplify irx-1 cDNA (1131 bp). irx-1 cDNA was cloned into pCR2.1 via TOPO TA cloning (Invitrogen), resulting in plasmid pSA17. The "irx-1 sense" construct pSA47 was cloned as follows: The pttr-39 promoter (composed of 891 bp immediately upstream of ttr-39) and irx-1 cDNA were PCR amplified and cloned into a derivative of pitr-1::DYS-1::YFP (kindly provided by Kang Shen, Stanford University, Stanford, CA) (Poon et al.,
2008) resulting in irx-1 sense RNA expression in GABA neurons. The unc-119 minigene [originally from MM051 (Maduro and Pilgrim, 1995)] was cloned into the backbone to produce pSA47. The "irx-1 antisense" construct pSA49 was cloned as follows: irx-1 cDNA was PCR amplified and inserted into pSA47 in the opposite orientation, replacing forwardirx-1 with reverse irx-1 sequence. The unc-119 minigene was replaced with punc-25::mCherry from pMLH41 (Earls et al., 2010) to result in pSA49. pSA47 (irx-1 sense) and pSA49 (irx-1 antisense) were linearized and ligated, and then transformed into unc-119 worms via microparticle bombardment as above. The presence of $i r x-1$ sense was identified by wild-type (unc-119+) movement, and the presence of irx-1 antisense was detected as mCherry expression in the GABA motor neurons.

\section{Electrophysiology}

Electrophysiology measurements were collected from dissected preparations, as previously described (Richmond and Jorgensen, 1999), with the following modifications to recording media. The extracellular solution consisted of the following (in mM): $150 \mathrm{NaCl}, 5 \mathrm{KCl}, 5 \mathrm{CaCl}_{2}, 4 \mathrm{MgCl}_{2}, 10$ glucose, 5 sucrose, 15 HEPES, pH 7.4, 340 mOsm. The patch pipette was filled with the following (in $\mathrm{mm}$ ): $120 \mathrm{KCl}, 20 \mathrm{KOH}, 4 \mathrm{MgCl}_{2}, 5$ $\mathrm{N}$-tris[hydroxymethyl]methyl-2-aminoethane-sulfonic acid, $0.25 \mathrm{CaCl}_{2}, 4$ $\mathrm{Na}_{2} \mathrm{ATP}, 36$ sucrose, 5 EGTA, pH 7.2, 315 mOsm. Electrophysiological measurements were performed in wild-type, unc-55, and unc-55; irx1(csRNAi) with the oxIs351 GABA channelrhodopsin transgene in the background. Recordings of GABAergic activity were collected from body wall muscles, patched in the whole-cell voltage-clamp mode at a holding potential of $-60 \mathrm{mV}$, in the presence of $10^{-4} \mathrm{M} \mathrm{D}$-tubocurare (dTBC) to block muscle stimulation from acetylcholine receptors.

\section{DD remodeling time course}

For the DD remodeling time course, NC2296 was used as the wild-type reference for irx-1(csRNAi) to eliminate the possibility of remodeling delays due to unc-119 rescue or mCherry expression. Separate groups of 100 gravid wild-type (NC2296) and irx-1 (csRNAi) (NC2297) adults were picked to single $60 \mathrm{~mm}$ plates and allowed to lay eggs for $1 \mathrm{~h}$ and then removed from the plate. The midpoint of the hour in which eggs were laid was considered to be $t=0$. Plates were incubated at $23^{\circ} \mathrm{C}$ throughout the time course. Beginning at $21 \mathrm{~h}$ after lay and for each hour thereafter, larvae were picked off wild-type and irx-1(csRNAi) plates and imaged (as above) on a Zeiss Axiovert microscope. The number of dorsal SNB-1::GFP puncta, number of GFP+ neurons (i.e., GABA neurons), and number of mCherry+ neurons [i.e., wild-type mCherry or irx1(csRNAi)] were recorded for six or more worms for each time point. Data were recorded from worms with mCherry in $\geq 50 \%$ of GABA $(\mathrm{GFP}+)$ neurons. Results were pooled from three separate time course experiments.

\section{Construction and analysis of irx-1(gf)}

To generate pttr-39::irx-1::GFP; unc-119+, the entire GFP-tagged irx-1 ORF from the irx-1::GFP fosmid was PCR amplified and cloned into pCR2.1 via TOPO-TA cloning to yield pSA64. The irx-1::GFP ORF was then cloned into pSA47 (see above) to yield pSA70. This construct was transformed into unc-119 worms via microparticle bombardment as above. Animals exhibited mosaic wdEx753[pttr-39::IRX-1::GFP; unc$119+]$ expression in DD and VD motor neurons.

\section{Results}

\section{UNC-55 prevents GABA motor neuron synaptic remodeling}

In C. elegans adults, sinusoidal motion depends on a signaling network in which cholinergic motor neurons alternately excite dorsal or ventral muscle as GABAergic innervation concurrently inhibits contraction on the opposite side (Schuske et al., 2004). GABAergic signaling is provided by the "D-type" class of motor neurons comprised of six DD and 13 VD motor neurons. All D-type motor neurons adopt a stereotypical morphology with a circumferential commissure linking single anteriorly directed processes in the ventral and dorsal nerve cords (Fig. $1 A, B$ ). In the adult, DD and VD motor neurons adopt opposite synaptic arrangements; DDs innervate dorsal muscles, whereas VDs synapse 

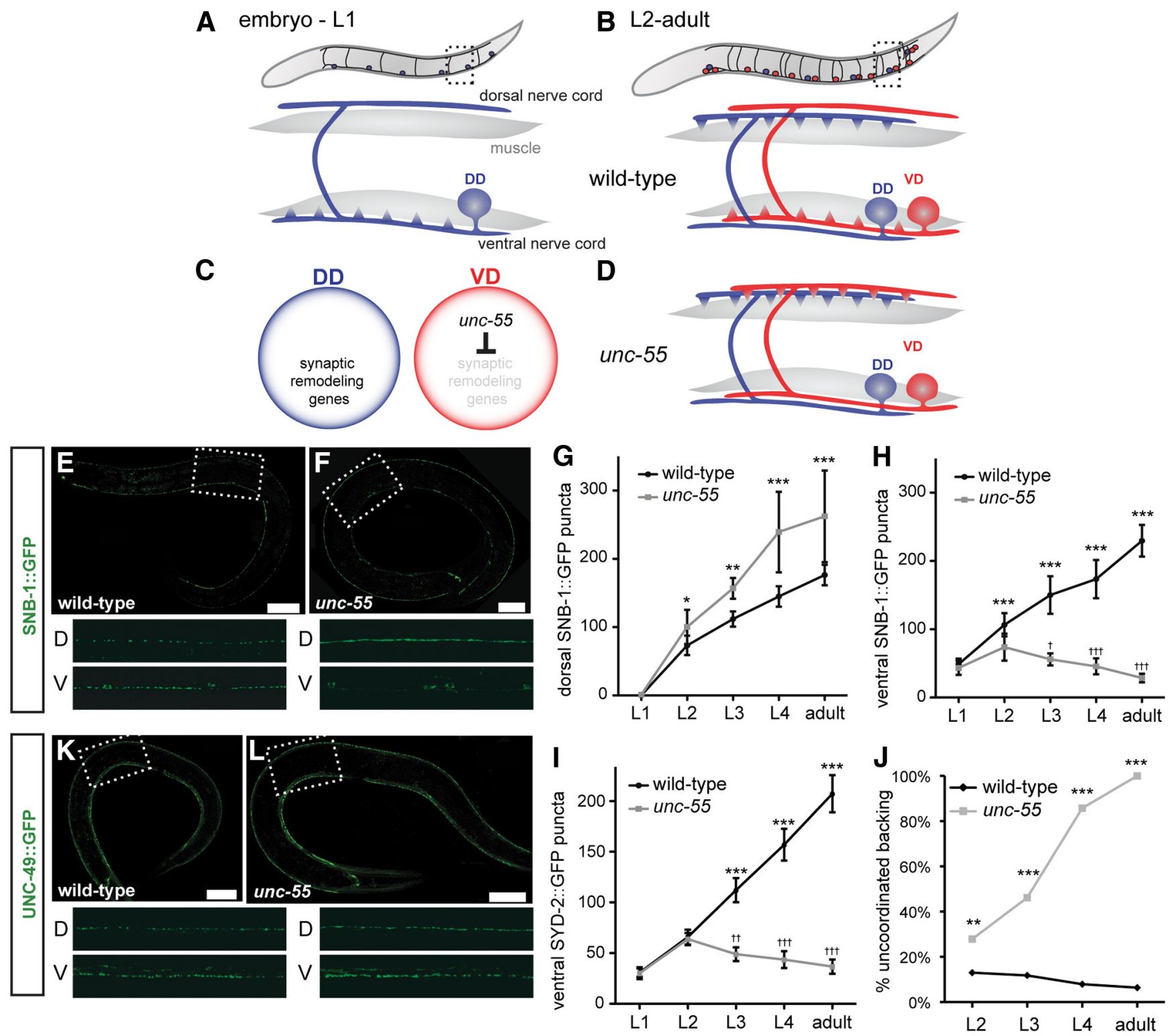

Figure 1. Synaptic remodeling in wild-type and unc-55 GABAergic motor neurons. For all panels, dorsal is up and anterior is to the left. $A$, Embryonic DD motor neurons (blue) extend an anterior ventral process and commissure to the dorsal nerve cord; NMJs are established with ventral muscles. $B$, Toward the end of $L 1$ larval period, ventral DD motor neuron NMJs are removed and new NMJs are assembled in the dorsal DD process. Postembryonic VD motor neurons (red) are born during this stage and adopt ventral NMJs. C, UNC-55 is selectively expressed in VD motor neurons to block synaptic remodeling. $\boldsymbol{D}$, VD motor neurons remodel ectopically in unc-55 mutants and adopt dorsal NMJs. $\boldsymbol{E}-\boldsymbol{H}$, The synaptic vesicle marker, SNB-1::GFP, marks GABAergic motor neuron NMJs. Scale bars: $20 \mu \mathrm{m}$; inset panels, $50 \mu \mathrm{m}$. E, SNB-1::GFP puncta are visible in both dorsal (DD motor neurons) and ventral (VD motor neurons) adult nerve cords. $F$, In unc-55 mutants, ventral SNB-1::GFP puncta are depleted, whereas the number of dorsal SNB-1::GFP puncta increases. $G, H$, Quantification of SNB-1::GFP puncta in dorsal and ventral nerve cords throughout development. $n \geq 10$ for each genotype at each stage. Error bars indicate SD. ${ }^{*} p<0.05,{ }^{* *} p<0.01,{ }^{* * *} p<0.001$ for wild-type versus unc-55 in two-way ANOVA with Bonferroni's multiple-comparison test. G, Dorsal SNB-1::GFP puncta are more numerous in unc-55 mutants than wild type due to ectopic remodeling of VD motor neurons. $\boldsymbol{H}$, Ventral SNB-1::GFP puncta are more numerous in wild type than in unc-55 mutants. Ventral SNB-1::GFP puncta are significantly depleted during development in unc-55 mutants, indicating that VD motor neurons initially establish ventral NMJs in the L2 and then remodel to favor dorsal outputs in the adult. ${ }^{\dagger} p<0.05,{ }^{+{ }^{+t}} p<0.001$, L2 versus $\mathrm{L} 3, \mathrm{~L} 4$, or adult for one-way ANOVA with Bonferroni's multiple-comparison test. I, Quantification of SYD-2::GFP puncta, which marks the presynaptic apparatus, in the ventral nerve cord throughout development. SYD-2::GFP localization in unc-55 is indistinguishable from wild type at the L2 stage, but ventral SYD-2::GFP puncta are depleted as VD motor neurons remodel during development. $n \geq 10$ for each genotype at each stage. Error bars indicate SD. ${ }^{* * *} p<0.001$ for wild-type versus unc-55 in two-way ANOVA with Bonferroni's multiple-comparison test; ${ }^{+t} p<0.01,{ }^{+t+} p<0.001$, L2 versus $\mathrm{L} 3$, L4, or adult for one-way ANOVA with Bonferroni's multiple-comparison test. J, VD motor neurons initially form ventral neuromuscular junctions in unc -55 as indicated by movement similar to wild type at younger stages, but we observe a progressive increase in uncoordinated movement as the animal ages and VD motor neurons remodel. ${ }^{* *} p<0.01,{ }^{* * *} p<0.001$ for wild-type versus unc-55, Fisher's exact test; $n \geq 100$ for each genotype at each stage. $\boldsymbol{K}, \boldsymbol{L}, \mathrm{GABA}$ receptor localization in muscle is not perturbed by VD motor neuron synaptic remodeling. GABA receptor (UNC-49::GFP) localization in body wall muscle $(\boldsymbol{K})$ is normal in unc-55 adults ( $\boldsymbol{L}$ ), suggesting that L2 unc-55 VDs initially form functional ventral synapses. Scale bars: $20 \mu \mathrm{m}$; inset panels, $50 \mu \mathrm{m}$.

with ventral muscles (White et al., 1976). This complementary pattern is established during the first larval stage by a developmentally regulated remodeling program. DD motor neurons are generated in the embryo and initially synapse with ventral muscles (Fig. $1 \mathrm{~A}$ ). At the end of the first larval stage (L1), ventral DD neuromuscular junctions (NMJs) are removed as new DD NMJs appear on the dorsal side. This apparent reversal in neuronal polarity is achieved within the existing DD neuron, which remains intact throughout the remodeling process (Fig. $1 B$ ). VD motor neurons are born at the end of the L1 stage and establish 
ventral NMJs that are maintained throughout the life of the animal (Fig. 1B) (White et al., 1978).

The single COUP-TF homolog in C. elegans, UNC-55, is initially expressed in L2 stage VD motor neurons but is not detected in DD motor neurons (Shan et al., 2005). Genetic analysis has suggested a model in which UNC-55 functions as a transcriptional switch to block the DD remodeling program (Fig. 1C); in unc-55 mutants, VD motor neurons adopt the dorsal output pattern of adult DD motor neurons (Fig. 1D) (Walthall and Plunkett, 1995). The consequent loss of VD-dependent inhibition of ventral muscles results in uncoordinated movement (i.e., ventral coiling). A negative regulatory role for UNC-55 in the DD remodeling program is also supported by the finding that forced expression of UNC-55 in DD motor neurons blocks the normal relocalization of synapses to the dorsal side in the L1 (Shan et al., 2005).

We confirmed the VD output defect in unc-55 mutants by visualizing GABAergic synapses with the GFP-tagged SNB-1/synaptobrevin marker juIs1 [punc-25::SNB-1::GFP] (Fig. 1E,F) (Hallam and Jin, 1998). As shown in Figure $1 G$, dorsal SNB-1::GFP puncta are more abundant in the adult unc-55 mutant than in wild type. This phenotype arises from the progressive remodeling of VD motor neurons throughout larval development; the number of dorsal GABAergic synapses increases from the L2 to the adult (Fig. 1G). Ventral GABAergic synapses are concurrently reduced during this period until the adult stage when few SNB-1::GFP puncta are observed in the ventral nerve cord of unc-55 animals (Fig. $1 \mathrm{H}$ ). To determine whether other presynaptic components are similarly relocated, we used the GFP-tagged SYD-2/liprin- $\alpha$ to mark the VD presynaptic membrane domain. At the L2 stage, the number of SYD-2::GFP ventral puncta in unc-55 animals is comparable with wild type but are progressively depleted during later larval stages in a pattern mimicking that of SNB-1::GFP (Fig. 1 I).

The initial ventral enrichment and then subsequent elimination of SNB-1::GFP and SYD-2::GFP in the unc-55 mutant suggests that VD motor neurons establish synapses in L2 larva, but that these ventral NMJs are gradually removed as development proceeds (e.g., $74 \pm 20$ SNB-1::GFP puncta in unc-55 L2 vs $29 \pm$ 6 in unc-55 adult). Because ventral output from VD motor neurons is required for normal locomotion, this model predicts that coordinated movement should progressively decline as unc-55 mutants undergo larval development. To test this idea, we quantified the Unc-55 backward movement defect at different larval stages. Although this experiment showed that a higher proportion of L2 larvae display uncoordinated backward locomotion than wild type ( $29 \%$ of $u n c-55$ vs $13 \%$ of wild-type), this disparity is enhanced during larval development until the adult stage when virtually all unc-55 mutant animals show uncoordinated backward movement versus only $6 \%$ of wild-type animals (Fig. $1 \mathrm{~J}$ ). This behavior parallels the progressive depletion of synaptic markers in the ventral nerve cord during unc-55 development (Fig. $1 \mathrm{H}, \mathrm{I}$ ) and therefore is consistent with the proposal that VD synapses are initially functional but are then removed as ectopic remodeling ensues.

As an additional test of the new finding that $\mathrm{VD}$ motor neuron initially establish ventral synapses in unc-55 mutants, we examined the localization of the postsynaptic GABA receptor UNC49B to ventral muscles, a result that depends on a synaptic signal from GABAergic motor neurons (Gally and Bessereau, 2003). UNC-49::GFP puncta are initially evident on the ventral side in early L1 larvae at locations corresponding to DD presynaptic markers and are normally retained throughout larval development. In a genetic mutant (lin-6) that blocks postembryonic cell divisions that give rise to VD motor neurons, these ventral UNC49B clusters are removed after the L2 stage. This result indicates that VD input is required to sustain ventral UNC-49B clusters after DD motor neurons remodel (Gally and Bessereau, 2003). Thus, our observation that ventral UNC-49::GFP puncta persist throughout larval development in an unc-55 mutant (Fig. $1 K, L$ ) is consistent with the conclusion that VD motor neuron synapses are initially established in L2 larvae as suggested by the coincident ventral localization of SNB-1 and SYD-2 presynaptic markers at this stage (Fig. $1 H, I$ ). Together, our findings argue that the Unc-55 synaptic defect in VD motor neurons arises from ectopic activation of a remodeling program that effectively dismantles an initial set of signaling competent synapses to reconstitute them on the dorsal side. Because this sequence of synaptic assembly and translocation in the VDs parallels that of DD motor neurons, we reasoned that the identification of unc-55 targets could reveal genes with key roles in both classes of remodeling neurons.

\section{Cell-specific expression profiles reveal candidate unc-55 target genes}

Because UNC- 55 has been shown to function as a negative regulator of gene expression (Shan et al., 2005), it follows that UNC- 55 targets should be enriched in a gene expression profile of GABAergic motor neuron transcripts from an unc-55 mutant compared with wild type in which remodeling gene expression is limited to DD motor neurons (Fig. 2A). We used the mRNA tagging method (Fig. 2B,C) (Roy et al., 2002; Von Stetina et al., 2007a,b; Spencer et al., 2011) to isolate GABAergic-specific transcripts at the mid-L2 stage when VD motor neuron remodeling is initiated in unc-55 mutants (Fig. $1 \mathrm{H}$ ). A total of 188 transcripts showed significant elevation ( $\geq 2$-fold; $<1 \%$ false discovery rate; see Materials and Methods) in the unc-55 mutant versus wildtype profiles, whereas only 11 genes were depleted relative to wild type (Table 1). This finding is consistent with the proposal that UNC-55 protein functions as a transcriptional repressor (Shan et al., 2005). Seventy-nine (42\%) of the 188 transcripts that are de-repressed in unc-55 mutant GABAergic motor neurons are conserved in humans (BLAST $<\mathrm{e}^{-10}$ ). Approximately one-half (88) of the candidate unc-55 targets are novel with uncharacterized functions. The balance of 100 unc-55-enriched candidate synaptic remodeling genes were classified into functional groups based on KOGs, BLAST homolog function, or other identifying information compiled on wormbase.org (release WS220) (Von Stetina et al., 2007b) (Table 1).

\section{An RNAi screen identifies unc-55 regulated targets that function as synaptic remodeling genes}

UNC-55 is proposed to prevent synaptic remodeling by repressing transcription of specific target genes (Fig. 1C) (Shan et al., 2005). This assumption predicts that genetic ablation of these unc-55 regulated genes should effectively "suppress" the Unc- 55 synaptic phenotype by disabling the ectopic remodeling process. Therefore, we used RNAi (see Materials and Methods) to screen candidate unc-55 targets (Table 1) for the restoration of GABAergic synapses to the ventral side of unc-55 mutants (Fig. $3 A-C$ ). Bacterial RNAi feeding clones for 138 of the 188 candidate unc-55 targets available in the Ahringer RNAi library (Kamath et al., 2003) were tested for induction of ventrally localized SNB-1::GFP puncta in RNAi-treated unc- 55 animals versus an empty vector negative control (see Materials and Methods) (Fig. 3D). This assay revealed 
A

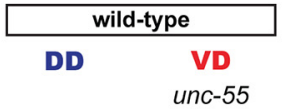

$\perp$

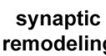

genes

$\mathbf{B}$
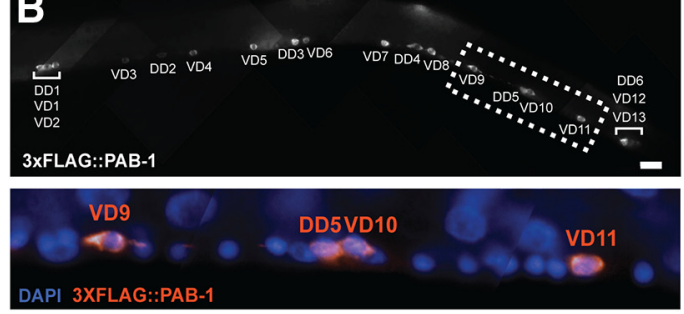

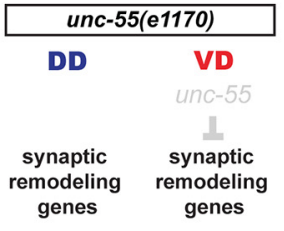

enes
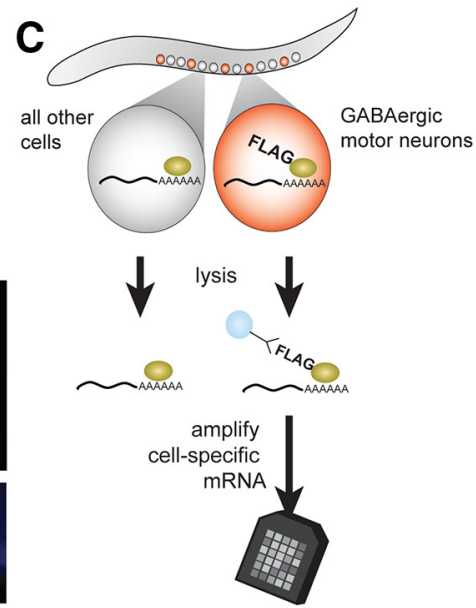

Figure 2. Identification of unc-55-regulated transcripts by cell-specific gene expression profiling. $\boldsymbol{A}$, Experimental design. In wild-type L2 larvae, synaptic remodeling genes are expressed in DD motor neurons but not in VD motor neurons; in unc-55(e1170) larvae, synaptic remodeling genes are also expressed in VD motor neurons. $B$, Anti-FLAG staining of FLAG-tagged PAB-1 transgenic line, wdls31[pttr-39::3xFLAG.:PAB-1] is restricted to GABAergic motor neurons in L2 stage larvae (top); ventral cord motor neuron soma are stained with DAPI (bottom). Scale bar, $10 \mu \mathrm{m}$. C, The mRNA tagging method. Lysates are treated with anti-FLAG-coated beads to capture FLAG-tagged PAB-1 cross-linked to cell-specific mRNA. Immunoprecipitated mRNA is amplified and labeled for hybridization to a C. elegans Affymetrix GeneChip array (see Materials and Methods).
73 of 76 wild-type DDs and 77 of 78 of unc-55 DDs). Moreover, IRX-1::GFP is not expressed in wild-type VD motor neurons (2 of 170) but is de-repressed in unc-55 VD motor neurons (171 of 176). Weak IRX-1::GFP expression was also observed in hypodermal nuclei and in two unidentified head neurons (data not shown). We found that the Pitx-1 homolog unc-30, which is necessary for DD remodeling in the $\mathrm{L} 1$, is also required for expression of IRX-1::GFP in GABA motor neurons (GFP expression in 0 of 40 DD motor neurons and 0 of $90 \mathrm{VD}$ motor neurons). This result is consistent with the idea that the GABAergic motor neuron synaptic remodeling program is controlled by modulation of the opposing roles of UNC-30 (positive) and UNC-55 (negative) (Shan et al., 2005). Together, these findings support a role for irx-1 in synaptic remodeling and confirm that the native ir $x$ - 1 gene is negatively regulated by unc-55. multiple suppressors of the Unc-55 phenotype, including the C-lectin clec-44, the Iroquois homeodomain-containing transcription factor $i r x-1$, the DEG/ENaC channel subunit $u n c-8$, the Arp $2 / 3$ subunit arx -5 , the Hedgehog-related ligand grl-4, and the synaptobrevin homolog snb-2 (Fig. $3 F$ ). In total, this screen detected significant RNAi suppression of the Unc-55 synaptic phenotype in 49 candidate $u n c-55$-regulated genes for a hit rate of $\sim 40 \%$. We note that 19 of these synaptic remodeling genes are conserved in humans (Fig. 3G, Table 1). Genes in our microarray profile that do not show RNAi suppression of the Unc-55 remodeling phenotype could be involved in other unc-55-dependent traits.

\section{ir $x$-1 is expressed in remodeling GABAergic motor neurons}

We selected the $i r x-1$ gene from the list of Unc- 55 suppressors for additional experiments to confirm its role in GABAergic synaptic remodeling. irx-1 encodes the single nematode homolog of the Iroquois homeodomain protein (Mukherjee and Bürglin, 2007). Iroquois has been previously shown to pattern neural tissue in other organisms (Cavodeassi et al., 2001; Gómez-Skarmeta and Modolell, 2002) and therefore offers an attractive opportunity to discover a new and potentially conserved role in synaptic remodeling.

The elevation of irx-1 transcript in unc-55 mutants suggests that $i r x-1$ is negatively regulated by $u n c-55$. Moreover, the RNAi results indicate that ir $x-1$ is required for GABA neuron remodeling. These findings suggest that ir $x-1$ should be normally expressed in remodeling DDs and de-repressed in unc-55 VDs (Fig. $1 C)$. To test this idea, we engineered an ir $x-1$ reporter gene in which GFP was fused to the irx-1 coding sequence in a construct that spans the complete $i r x-1$ gene as well as large flanking regions (see Materials and Methods). The resultant IRX-1::GFP fusion protein is capable of rescuing the lethal phenotype of an irx-1 null allele (data not shown). Thus, we reasoned that endogenous ir $x-1$ gene regulatory sequences should be preserved in this construct and that the chimeric IRX-1::GFP protein retains native activity. IRX-1::GFP was consistently detected in the nuclei of DD motor neurons from both wild-type and unc-55 mutant animals (Fig. 4,

\section{the Unc-55 backward locomotion defect \\ Ectopic ir $x$ - 1 function is required for} In unc-55 mutants, the loss of GABAergic ventral input from VD motor neurons results in ventral coiling during backward locomotion (Walthall and Plunkett, 1995). If irx-1 function is required for VD synaptic remodeling in an $u n c-55$ mutant, then the loss of ir $x-1$ activity should preserve ventral VD inputs and thus restore normal backward movement (i.e., suppress the Unc-55 movement defect). Because an irx-1 null allele results in embryonic and early larval lethality, we used global RNAi to test this model. As expected, unc-55 animals from control RNAi plates displayed the characteristic backward Unc phenotype (Fig. 5A). In contrast, a substantial fraction of surviving unc-55 animals from ir $x$-1 RNAi feeding plates were capable of sustained backward locomotion (Fig. 5B). We quantified this effect by scoring three distinct categories of movement: severe Unc (coils ventrally with tap on the head), moderate Unc (attempts backward locomotion before coiling ventrally), and wild type (executes at least two body bends of backward locomotion; see Materials and Methods). All control-treated unc-55 adults exhibited Unc backward locomotion (39\% severe Unc, $61 \%$ moderate Unc; $N=61$ ); in contrast, most of the irx-1 RNAi-treated unc-55 adults showed wild-type backward locomotion ( $80 \% ; N=50)$ (Fig. $5 B)$. The small fraction showing some Unc behavior ( $18 \%$ moderate Unc, $2 \%$ severe Unc) may be attributed to the incomplete effect of RNAi knockdown or to necessary remodeling roles for additional unc-55 regulated genes (Fig. $5 B$ ). To confirm that ir $x-1$ function in this pathway is cell autonomous, we constructed a transgenic line carrying a heritable GABA-specific irx-1 RNAi array, $w d E x 686[i r x-1$ (csRNAi)]. The irx-1 (csRNAi) transgene expresses both sense and antisense irx-1 RNA strands under control of the GABA-specific ttr-39 promoter; GABA neurons with the irx1 (csRNAi) array are marked by coexpression of GABA::mCherry (see Materials and Methods). These experiments confirmed that selective ablation of irx-1 in ventral cord GABA neurons is sufficient to suppress the Unc-55 backward movement defect (data not shown). 
Table 1. Candidate synaptic remodeling genes identified by microarray analysis and RNAi suppression screen

\begin{tabular}{|c|c|c|c|c|c|}
\hline Protein type & $\begin{array}{l}\text { Enriched in } \\
\text { microarray }^{a}\end{array}$ & $\begin{array}{l}\text { Screened for Unc-55 } \\
\text { suppression }\end{array}$ & Hits $^{b}$ & Conserved hits $^{c}$ & Gene names \\
\hline Channels and membrane proteins & 11 & 9 & 4 & 2 & unc-8, F28H1.4 \\
\hline Secreted surface protein & 6 & 4 & 1 & 0 & \\
\hline Hedgehog-like family & 4 & 3 & 1 & 1 & $g r l-4$ \\
\hline G-protein signaling & 2 & $3^{d}$ & 2 & 1 & cnt-1 \\
\hline Fibronectin & 3 & 2 & 0 & 0 & \\
\hline Lectin & 2 & 1 & 1 & 1 & clec-44 \\
\hline Transcriptional control & 5 & 4 & 2 & 1 & $i r x-1$ \\
\hline Cytoskeleton & 2 & 2 & 1 & 1 & $\operatorname{arx}-5$ \\
\hline Lipid binding & 3 & 3 & 1 & 0 & \\
\hline Calcium binding & 3 & 3 & 0 & 0 & \\
\hline Iron binding & 3 & 0 & 0 & 0 & \\
\hline FGF signaling & 2 & 2 & 1 & 1 & $T 23 B 12.5$ \\
\hline Neuro-related & 2 & 2 & 1 & 1 & snb-2 \\
\hline Ubiquitin & 1 & 1 & 0 & 0 & \\
\hline Peptidase inhibitor & 5 & 5 & 1 & 0 & \\
\hline Metalloprotease & 2 & 2 & 2 & 2 & nas-38, T19D2.1 \\
\hline Other enzyme & 24 & 19 & 9 & 4 & C11E4.1, F20G2.1, K01A2.5,ZC434.9 \\
\hline Collagen & 14 & 11 & 3 & 3 & col-12, col-54, col-77 \\
\hline Other & 6 & 1 & 0 & 0 & \\
\hline Novel/uncharacterized & 88 & 61 & 19 & 1 & pqn-36 \\
\hline Total & 188 & 138 & 49 & 19 & \\
\hline
\end{tabular}

${ }^{a}$ Determined by at least twofold enrichment, $1 \%$ false discovery rate.

${ }^{b}$ Determined by significant enrichment of ventral SNB-1::GFP puncta and a phenotype observed in at least $50 \%$ of animals screened. See Materials and Methods.

'Determined by BLAST score of $<10^{-10}$.

${ }^{d}$ ArfGAP cnt- 1 added to candidate target RNAi screen based on its enrichment in a dataset with less stringent SAM parameters ( $>1.5 \times$ enrichment, $5 \%$ false discovery rate).

\section{irx-1 promotes synaptic remodeling in unc-55 mutant VD motor neurons}

Our finding that cell-specific irx-1 RNAi restores backward locomotion to an unc-55 mutant suggests that cell-autonomous knockdown of $i r x-1$ is also likely to block synaptic remodeling in unc-55 VD motor neurons. To test this prediction, we scored the distribution of SNB-1::GFP puncta in the dorsal and ventral nerve cords of unc-55; irx-1 (csRNAi) mutant animals. These arrays show mosaic expression and the mCherry marker identifies the subset of GABAergic neurons that actually carry either the irx-1(csRNAi) or the $w d E x 658$ control array (see Materials and Methods). We first confirmed that SNB-1::GFP is strongly enriched in the dorsal nerve cord (Fig. 6A) and depleted in the ventral nerve cord (Fig. 6C) of mCherry-marked GABA motor neurons in unc-55 mutant animals carrying the $w d E x 658$ control array. In contrast, $u n c-55$ mutant VD motor neurons that express irx-1(csRNAi) show fewer SNB-1::GFP puncta in the dorsal nerve cord (Fig. 6B) with a concomitant increase in ventral SNB-1::GFP-marked NMJs (Fig. 6D, arrowheads). To quantify this effect, we counted SNB-1::GFP puncta in a region (VD anterior region) that includes the dorsal and ventral processes of the given mCherry marked VD motor neuron (Fig. 6E). In wild-type animals, the number of ventral NMJs (from VDs) was roughly equivalent to the number of dorsal NMJs (from DDs) in each VD anterior region (Fig. 6F; $12.7 \pm 2.9$ ventral puncta, $10.1 \pm 2.8$ dorsal puncta, $N=50 \mathrm{VD}$ anterior regions). As expected, in unc-55 mutants, the number of dorsal SNB-1:: GFP puncta was significantly increased $(22.4 \pm 4.0 ; p<0.0001$ vs wild type, two-way ANOVA with Bonferroni's multiplecomparison test) with a corresponding depletion of ventral puncta $(2.3 \pm 1.7 ; p<0.0001$ vs wild type, $N=50 \mathrm{VD}$ anterior regions) (Fig. 6F). Compared with unc-55 mutant VD motor neurons, unc-55; irx-1(csRNAi) VDs had significantly fewer dorsal puncta and more ventral puncta (Fig. $6 F ; 14.6 \pm 2.5$ dorsal puncta, $p<0.0001$ vs $u n c-55 ; 12.9 \pm 2.3$ ventral puncta, $p<$
0.0001 vs $u n c-55 ; N=50 \mathrm{VD}$ anterior regions). These results confirm that knockdown of irx-1 specifically in unc-55 mutant VDs prevents remodeling and substantiates the proposal that derepression of $i r x-1$ in $u n c-55$ mutants promotes VD remodeling.

\section{Cell-specific ir $x$ - 1 knockdown restores GABAergic input to ventral muscles in unc-55 mutants}

The restoration of backward locomotion (Fig. 5) as well as ventral GABAergic SNB-1::GFP puncta to unc-55; irx-1 (csRNAi) animals (Fig. 6) is indicative of functional GABAergic inputs to ventral body wall muscles. To test this idea, we recorded miniature postsynaptic events (mPSCs) in the ventral muscles of wild type, unc55, and unc-55; irx-1(csRNAi). These animals were treated with the cholinergic antagonist $\mathrm{dTBC}$ to insure that all mPSCs are exclusively induced by GABAergic signaling (Richmond and Jorgensen, 1999). Recordings from ventral muscles detected robust mPSCs in wild-type adults (Fig. 7A). In contrast, mPSCs were rarely observed in ventral recordings of $u n c-55$ mutants (Fig. $7 A$ ) as predicted from the observation that VD synapses are largely confined to the dorsal nerve cord in $u n c-55$ animals (Fig. $1 D, F$ ) (Walthall and Plunkett, 1995). irx-1(csRNAi) expression in unc-55 mutants restores mPSCs to ventral muscle (Fig. 7A) and thus indicates that the ventral GABAergic SNB-1::GFP puncta that reappear in this strain (Fig. 6D,F) correspond to functional synapses. These results were confirmed by quantification; mPSCs were virtually absent from unc-55 ventral muscles $(45.8 \pm 20.3$ events/s in wild type, $N=5$, vs $0.05 \pm 0.06$ events/s in unc-55, $N=4 ; p<0.02$, Mann-Whitney $U$ test) but were significantly restored in unc-55; irx-1(csRNAi) animals $(22.6+15.0$ events/s, $N=6, p<0.02$, vs unc-55, $p>0.1$ vs wild type, Mann-Whitney $U$ test). Robust ventral mPSCs were detected in $u n c-55$ mutants in the absence of dTBC, thereby demonstrating that ventral cholinergic inputs are not perturbed by ectopic VD remodeling (Fig. $7 B)$. Similar mPSC event amplitudes were observed for all genotypes ( $p<0.01$ in all comparisons, Mann-Whitney $U$ test), sug- 
A

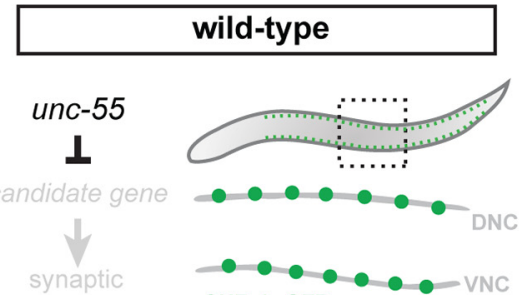

remodeling SNB-1::GFP

B

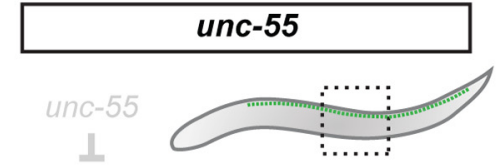

candidate gene

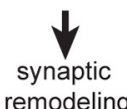

C remodeling

\section{unc-55; candidate RNAi}

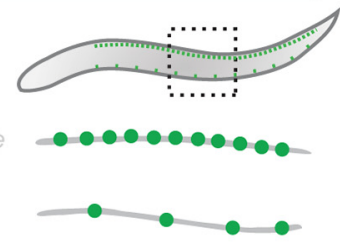

wild-type; empty vector RNAi
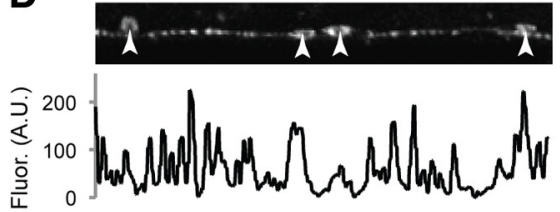

F unc-55; clec-44 RNAi

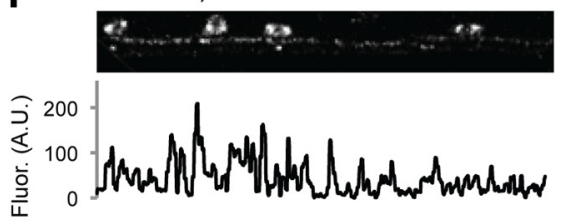

unc-55; unc-8 RNAi

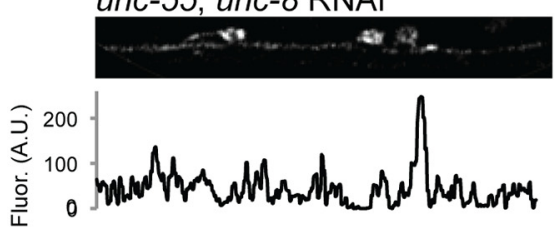

unc-55; grl-4 RNAi

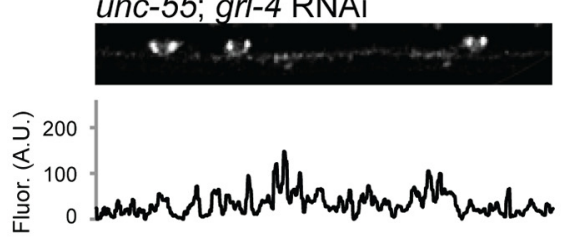

E unc-55; empty vector RNAi

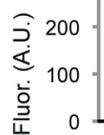

unc-55; irx-1 RNAi

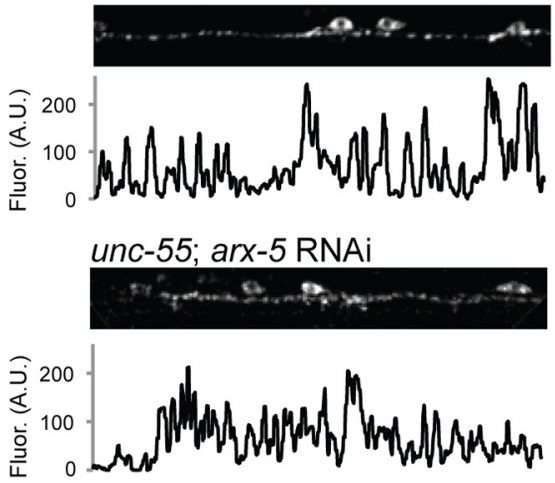

\section{unc-55; snb-2 RNAi}

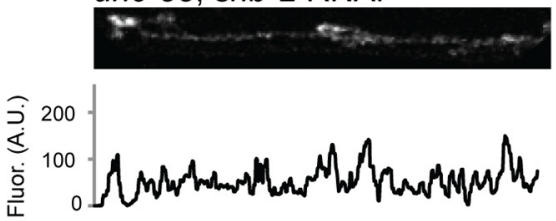

G

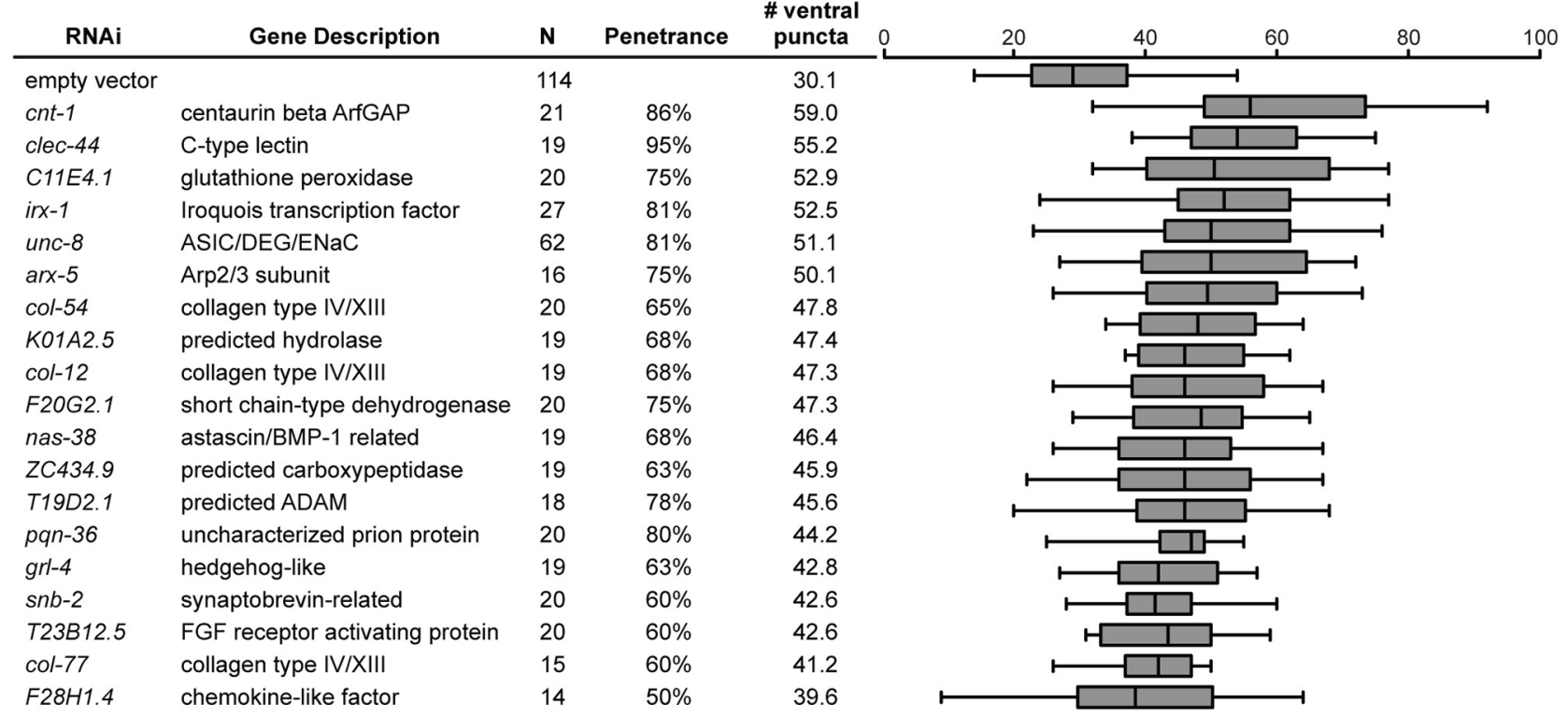

Figure 3. RNAi of candidate UNC-55 targets identifies genes required for synaptic remodeling. $A-C$, Experimental design of Unc- 55 suppression screen. $A$, In wild-type animals, SNB-1::GFP puncta (marking GABAergic NMJs) localize to both dorsal and ventral nerve cords. $\boldsymbol{B}$, In unc-55 animals, synaptic remodeling genes are de-repressed in VD motor neurons and SNB-1::GFP is depleted from the ventral nerve cord. C, RNAi knockdown of UNC-55 target genes in unc-55 mutants disrupts synaptic remodeling and restores SNB-1::GFP puncta to the ventral nerve cord. $D-F$, Representative views ( $50 \mu \mathrm{m}$ length) of GABAergic SNB-1::GFP in adult ventral nerve cords. Line scans (in arbitrary fluorescence units) of the ventral nerve cord (excluding DD/VD cell bodies; $\boldsymbol{D}$, arrowheads). $\boldsymbol{D}$, SNB-1::GFP puncta are abundant in the ventral nerve cord of wild-type adults $(\boldsymbol{E})$ but largely absent from unc-55(e1170) mutant animals treated with control RNAi (empty vector). $\boldsymbol{F}$, RNAi knockdown of candidate unc-55 targets (clec-44, irx-1, unc-8, arx-5, grl-4, snb-2) in unc-55 mutants partially restores ventral SNB-1::GFP puncta. G, Conserved hits from Unc-55 RNAi suppression screen. Hits were scored as significantly different from empty vector control ( $p<0.01$, Student's $t$ test) with restoration of ventral SNB-1::GFP puncta in $\geq 50 \%$ of animals scored. N represents all animals scored, combined from at least two separate RNAi treatments (see Materials and Methods). The boxes on the box-and-whisker plot span the 25th to 75 th percentile, and the whiskers indicate the minimum/maximum number of ventral puncta observed; the vertical line in each box indicates the 50 th percentile. Conserved hits show $<\mathrm{e}^{-10}$ versus human homologs by BLAST.

gesting that quantal neurotransmitter release for the residual mPSCs in unc-55 mutants and receptor sensitivity are unchanged (Fig. $7 C$ ). We conclude that the sparse occurrence of ventral mPSCs in unc-55 is due to a loss of ventral GABAergic input and that the restoration of ventral activity by irx-1(csRNAi) indicates reestablishment of ventral GABAergic synapses. Thus, these results confirm that $i r x-1$ activates a remodeling program in unc- 55 mutant GABA neurons that dismantles functional (ventral) synapses. 
A
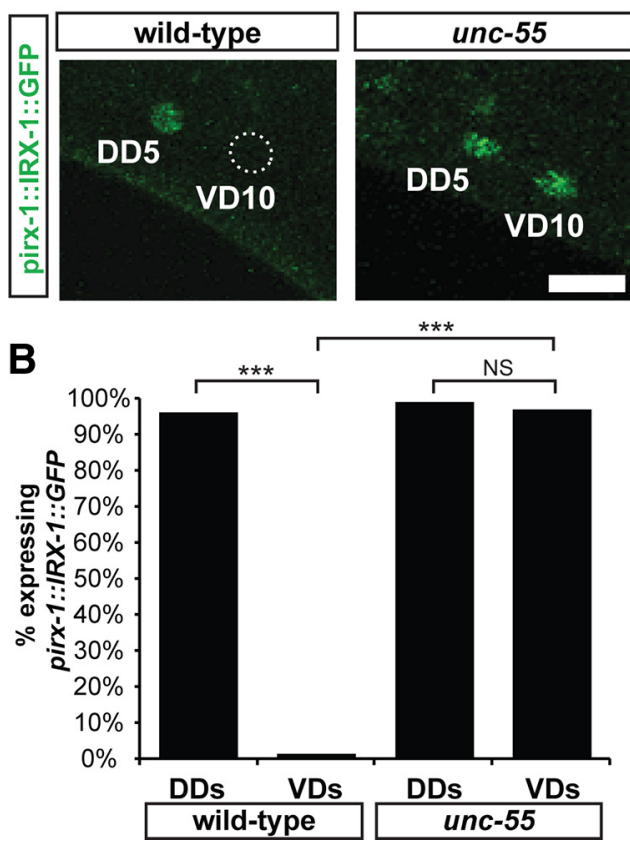

Figure 4. irx-1::GFP is expressed in remodeling GABAergic motor neurons. $A$, IRX-1::GFP is restricted to the nucleus of DD motor neurons in wild-type L4 larvae. In unc-55 mutants, IRX-1::GFP is observed in both DD and VD motor neurons (DD5 and VD10 shown for examples). Similar expression patterns were observed for all DD and VD motor neurons. Scale bar, $5 \mu \mathrm{m}$. $\boldsymbol{B}$, Quantification of IRX-1::GFP expression in GABAergic motor neurons of $L 2$ larvae. Note that IRX-1::GFP is de-repressed in unc-55 VD motor neurons. ${ }^{* * *} p<0.0001$; NS, not significant; Fisher's exact two-tailed test.

\section{ir $x-1$ is required for normal DD motor neuron remodeling}

Our results have shown that irx-1 is required for ectopic remodeling of VD motor neurons in unc-55 mutants. Because irx-1 is normally expressed in DD motor neurons, we next asked whether irx-1 is also necessary for DD remodeling. Dorsal synapses from DDs are established late in the first larval stage (White et al., 1978; Hallam and Jin, 1998). To visualize this remodeling event, we monitored the appearance of dorsal SNB-1::GFP puncta at $1 \mathrm{~h}$ intervals during this period (Fig. $8 \mathrm{~A}$ ). In wild-type larvae, dorsal SNB-1::GFP puncta are initially detected at $22 \mathrm{~h}$ (elapsed time since egg laying; see Materials and Methods) and increase in number until reaching a plateau at the onset $(\sim 30 \mathrm{~h})$ of the L2 stage. DD remodeling is significantly slower in L1 larvae carrying the $\operatorname{irx}-1$ (csRNAi) transgene with a $\sim 4$ h delay in the initial detection of dorsal SNB-1::GFP puncta at $26 \mathrm{~h}$ [Fig. $8 A{ }^{* *} p<0.01$, wild type vs $\operatorname{irx}-1$ (csRNAi), Student's $t$ test]. This effect is strikingly evident in DD motor neurons with high irx-1(csRNAi) expression (indicated by punc-25::mCherry; Fig. $8 C, D$, DD5) in which dorsal SNB-1::GFP puncta are small and few in number compared with DDs without cell-specific irx-1 knockdown (Fig. $8 C, D, \mathrm{DD} 6)$.

We note, however, that DD remodeling is not completely blocked by irx-1 (csRNAi) but eventually reaches completion later in larval development (Fig. $8 \mathrm{~A}$ ). To determine whether this effect could be due to an overall delay in the GABA neuron developmental program, we monitored the expansion of GABA motor neurons during this period. L1 larvae are born with 6 DD motor neurons; 13 VD motor neurons are added to the ventral cord by the end of the L1 stage (White et al., 1978). We noted no difference in rate of appearance of GABAergic motor neurons in wildtype versus irx-1(csRNAi) animals and conclude that the timing of this expansion is not perturbed by irx-1(csRNAi) [Fig. $8 B$; all time points $p>0.05$, wild type vs irx-1(csRNAi), Student's $t$ test]. We also confirmed that irx-1 RNAi does not inhibit expression from the $u n c-25$ promoter and therefore excluded the possibility that this would account for the reduced number of GFP puncta arising from the punc-25::SNB-1::GFP reporter (data not shown). On the basis of these results, we conclude that irx-1 normally promotes DD remodeling. However, because RNAi knockdown of ir $x-1$ retards but does not arrest DD remodeling, we conclude that additional genes functioning in parallel to ir $x-1$ may provide partially redundant function.

\section{irx-1 is sufficient to induce synaptic remodeling in VD motor neurons}

The finding that ir $x-1$ promotes DD remodeling suggested that ir $x$ - 1 might also be sufficient to drive synaptic remodeling in wild-type (i.e., unc-55+) VD motor neurons. To test this idea, we used the $t$ tr-39 promoter to express of IRX-1::GFP in GABAergic motor neurons. $t$ tr-39 is specific to DD and VD motor neurons (Cinar et al., 2005) and is not regulated by unc-55 (data not shown). IRX-1::GFP in these animals localizes to the nucleus (Fig. 9A) (see also Fig. 4A) of DDs and VDs, unlike the marker SNB-1::GFP, which remains in the cytoplasm. We used this difference in GFP localization to distinguish gain-of-function IRX-1::GFP-positive or irx-1 (gf) VDs (nuclear GFP) from wildtype VDs (cytoplasmic GFP only). Wild-type VD motor neurons in these mosaic animals show SNB-1::GFP puncta in the ventral process of the VD anterior region as expected (Fig. 9A, $B$, VD5). In contrast, $i r x-1(g f)$ VD motor neurons exhibit an Unc-55-like phenotype with excess dorsal SNB-1::GFP puncta coupled with a concomitant depletion on the ventral side (Fig. 9A, B, VD6). We quantified this result by counting SNB-1::GFP puncta adjacent to wild-type and irx-1(gf) VD motor neurons (Fig. 9C). Comparable numbers of dorsal (from DD motor neurons) and ventral (VD motor neurons) SNB-1::GFP puncta were detected in regions anterior to wild-type VDs (Fig. 9C; $10.2 \pm 2.4$ dorsal puncta vs $11.2 \pm 1.8$ ventral puncta; $N=20$ wild-type $\mathrm{VD}$ anterior regions). Ventral puncta were virtually eliminated, however, from irx-1 (gf) VDs, which show a strong Unc-55-like phenotype $[17.0 \pm 2.0$ dorsal puncta vs $1.7 \pm 1.7$ ventral puncta; $N=20$ $\operatorname{irx}-1(g f)$ VD anterior regions; ${ }^{* *} p<0.01$, two-way ANOVA with Bonferroni's multiple-comparison test]. The similarity of the cell-specific irx-1 (gf) phenotype to that of unc-55 suggests that ectopic ir $x-1$ is sufficient to induce synaptic remodeling in VD motor neurons and that ir $x-1$ is a primary downstream effector of the Unc-55 remodeling phenotype (Fig. 9D).

\section{Discussion}

Neural circuits may be dramatically reorganized during development by processes that remove existing synapses while creating new ones at alternative locations (Tissot and Stocker, 2000; Ding et al., 2007; Hashimoto et al., 2009). Synaptic remodeling is also actively initiated in the adult nervous system by external stimuli that trigger learning and memory (Feldman, 2009). In both contexts, transcription factors have been shown to fulfill essential roles in the remodeling process (Flavell et al., 2008; Boulanger et al., 2011). In C. elegans, the UNC-55 COUP-TF transcription factor controls a remodeling program that reorganizes the placement of synaptic outputs in GABAergic motor neurons. We used a cell-specific profiling strategy to identify candidate UNC-55-regulated transcripts and an RNAi screen to detect the subset of downstream targets that are required for synaptic remodeling. Our approach revealed a large and diverse array of synaptic remodeling genes with apparent homologs 
A

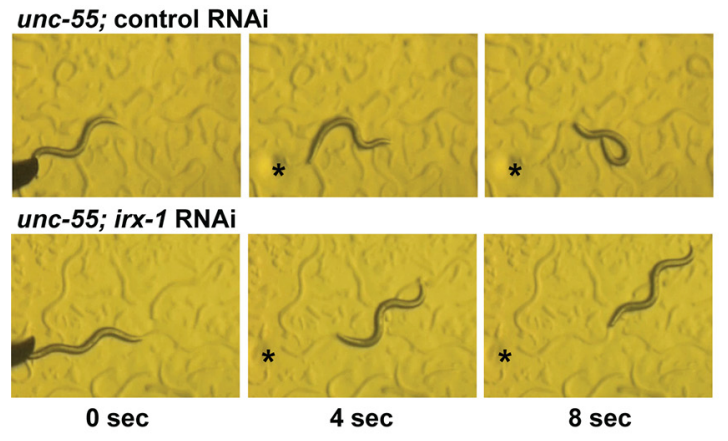

B

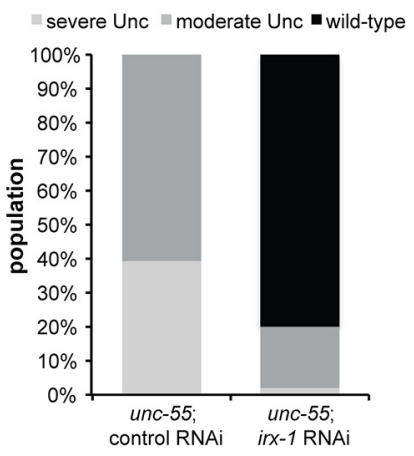

Figure 5. irx- 1 knockdown restores backward locomotion in unc-55 mutants. $A$, unc- 55 mutants coil ventrally during backward locomotion due to loss of inhibitory GABAergic input to ventral muscles (top panels). Treatment of unc- 55 mutants with irx-1 RNAi restores backward locomotion (bottom panels). Animals were tapped on the head (asterisk) at $t=0$. B, Quantification of irx- 1 suppression of backward movement defect in unc-55 adults treated with control (empty vector) or with irx-1 RNAi. Animals were binned in three categories: severe unc, which coil ventrally immediately after a head tap; moderate unc, which attempt backward locomotion but stop after coiling ventrally (for example, see top panels in $A$ ); and wild type, which sustain backward locomotion (at least 1 body length; for example, see bottom panels in $\boldsymbol{A}$ ). All control-treated animals exhibit uncoordinated backward locomotion $(N=61)$, whereas most $(>80 \%)$ irx-1-treated animals show wild-type backward movement $(N=50)$.
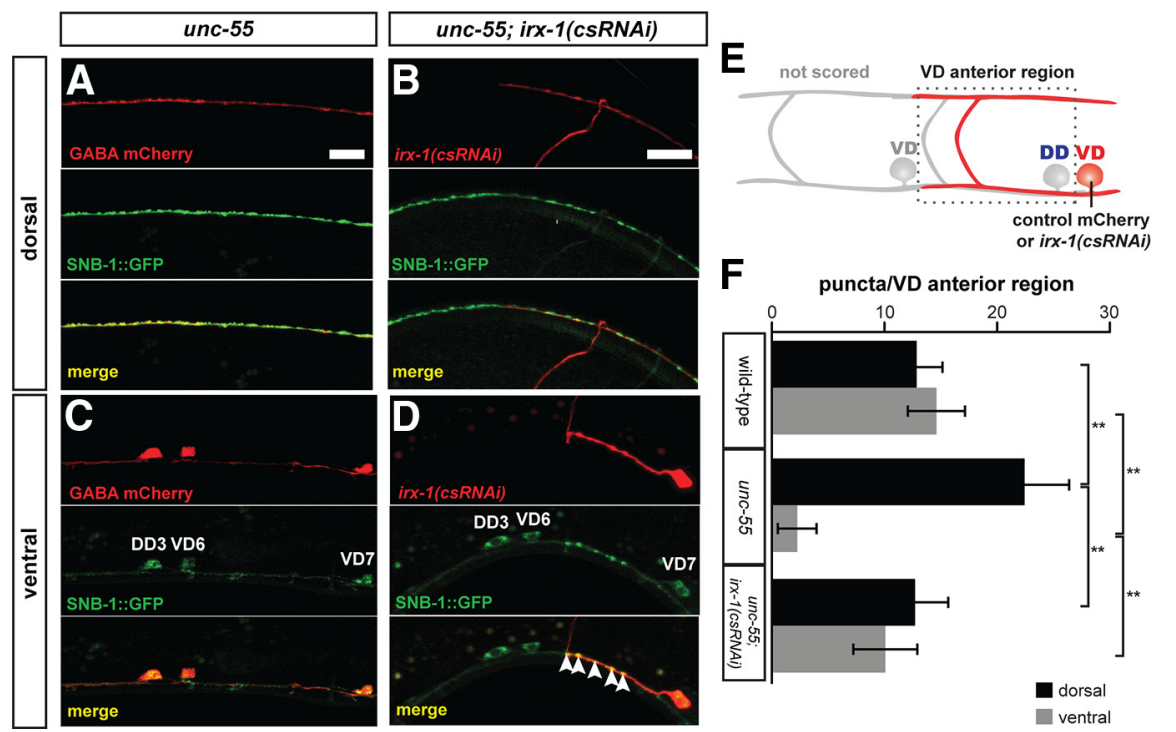

Figure 6. Cell-autonomous expression of irx-1 is required for synaptic remodeling in unc-55 VD motor neurons. $\boldsymbol{A}-\boldsymbol{D}$, SNB-1::GFP puncta label GABAergic NMJs. mCherry marks cells expressing the control wdEx658 transgenic array $(\boldsymbol{A}, \boldsymbol{C})$ or specific

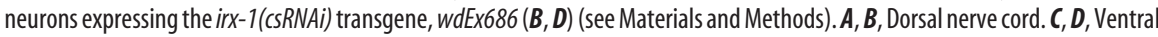
nerve cord. Common region of single adult animal in $\boldsymbol{A}$ and $\boldsymbol{C}$, and separate adult in $\boldsymbol{B}$ and $\boldsymbol{D}$. Scale bars, $10 \mu \mathrm{m}$. $\boldsymbol{A}$, Note dense array of SNB-1::GFP puncta in the dorsal nerve cord of unc-55 adult. $\boldsymbol{B}$, Fewer SNB-1::GFP puncta are observed in the region corresponding to dorsal arm of irx-1(csRNAi) expressing VD motor neuron (marked with mCherry). Note that dorsal SNB-1::GFP puncta from DD3 in this region do not overlap with the VD6 mCherry marker. C, SNB-1::GFP puncta are depleted from the ventral nerve cord of unc-55 adults. $\boldsymbol{D}$, Expression of irx-1(csRNAi) in VD7 (marked with mCherry) of an unc-55 mutant restores ventral SNB-1::GFP puncta. SNB-1::GFP puncta are absent from neighboring VD6 motor neuron that does not express irx-1(csRNAi). $\boldsymbol{E}$, The dotted lines denote anterior regions of mCherry-marked VD motor neurons in which SNB-1::GFP puncta were counted (see Materials and Methods). $\boldsymbol{F}$, Quantification of irx-1(csRNAi) suppression of the Unc-55 synaptic remodeling defect. ${ }^{* *} p<0.0001$, two-way ANOVA with Bonferroni's multiple-comparison test. $N=50$ VD anterior regions per genotype.

in humans. To validate this strategy and to establish experimental methods for systematic analysis of these targets, we defined the role for one of these genes, the conserved homeodomain-containing transcription factor, IRX-1/Iroquois, in synaptic remodeling. Our results show that IRX-1 is both necessary and sufficient in VD motor neurons for the Unc-55 remodeling phenotype. We also demonstrated that IRX-1 contributes to developmentally regulated remodeling of DD motor neurons. Thus, these results are consistent with the hypothesis that the ectopic remodeling activated in unc-55 mutant VD motor neurons includes key components that also drive the reorganization of synaptic output in DD motor neurons. We note, however, that DD remodeling is delayed but not entirely prevented when $i r x-1 /$ Iroquois function is disabled (Fig. 8A), whereas remodeling of unc-55 mutant VDs is completely blocked (Fig. 6). This finding suggests that additional partially redundant synaptic remodeling genes that function in parallel to irx-1 may be active in DD motor neurons (Fig. 9D). The existence of DD-specific remodeling genes could also explain why our RNAi screen was capable of detecting transcripts with roles in ectopic VD remodeling but did not prevent DD motor neurons from eventually establishing dorsal synapses in the adult (Fig. 3).

\section{The COUP-TF homolog unc-55} functions as a transcriptional switch to block an irx-1-dependent synaptic remodeling program

unc-55 encodes the single COUP-TF nuclear hormone receptor in C. elegans (Zhou and Walthall, 1998). COUP-TF proteins function as transcription factors and adopt multiple roles in neural development including the widely observed function of preventing the adoption of alternative cell fates (Armentano et al., 2007; Tomassy et al., 2010). For example, in Drosophila, seven-up/ COUP-TF preserves the specification of photoreceptors R1, R3, R4, and R6 by blocking the R7 fate (Mlodzik et al., 1990), a role that resembles that of unc-55, which prevents VD motor neurons from expressing a specific DD motor neuron trait (Walthall and Plunkett, 1995). Similarly, COUP-TFI and COUP-TFII maintain the balance of GABAergic neuron types arising from different regions of the ganglionic eminence in the mammalian forebrain (Lodato et al., 2011). The conservation from nematodes to mammals of analogous roles for COUP-TFs in establishing GABAergic neuron fates is intriguing and may reflect a primordial genetic program that operates in both contexts (Hammock et al., 2010).

Our results show that irx-1 is normally expressed in DDs to promote synaptic remodeling but is negatively regulated in nearby VDs by unc-55 (Fig. 4) to prevent that adoption of the DD pattern of synaptic output (Fig. 6). The ir $x$ - 1 locus encodes a transcription factor containing a homeodomain and flanking IRO box (Bürglin, 1997) with strong homology to human Iroquois genes. In other organisms, the Iroquois family of transcription factors has been shown to pattern the nervous system, with early roles in specification of neural ectoderm and a later function to refine neural architecture (Cavodeassi et al., 2001; Gómez-Skarmeta and Modolell, 2002). We 
report a new role for the C. elegans member of Iroquois family in a synaptic remodeling program that is antagonized by unc-55. Although COUP-TF and Iroquois proteins have been separately observed to perform developmental functions in common neural tissues (Matsumoto et al., 2004; Cheng et al., 2005; Kim et al., 2009; Tang et al., 2010), our results identify the first regulatory link between these two conserved transcription factors.

\section{Synaptic remodeling is a complex process requiring a variety of cellular pathways}

Our findings indicate that irx-1 coordinates the overall remodeling scheme by directing the elimination of ventral VD synapses while simultaneously promoting the creation of dorsal VD synapses (Figs. $6,9)$. This central role for irx-1 could result from its function as a master regulator that coordinates expression of separate sets of genes with remodeling roles at either dorsal or ventral synaptic locations. Alternatively, remodeling could be orchestrated by a cell-biological mechanism in which assembly of dorsal synapses activates a negative-feedback loop that destabilizes ventral synapses (Ding et al., 2007; Park et al., 2011). Our results do not distinguish between these possibilities, but systematic identification of irx-1 targets and their sites of action should help to resolve this question.

We have identified at least 49 additional genes that promote synaptic remodeling (Table 1). Whether these remodeling genes are also required for the creation of dorsal synapses awaits additional detailed analysis by genetic methods used here to assess the role of irx-1. This approach will be crucial for developing models of how these downstream effectors control remodeling. Nineteen of these genes encode proteins with conserved human homologs and together embody a wide array of potential cellular functions (Fig. $3 F, G$ ). This finding parallels profiling results for the transcription factor Mef2, which also identified a diverse spectrum of downstream genes that affect synaptic assembly in the hippocampus (Flavell et al., 2008). For example, the Arp2/3 component, arx -5 , is required for VD remodeling (Fig. $3 F, G)$. The Arp $2 / 3$ complex is composed of seven conserved components that function together to nucleate the formation of branched actin networks (Machesky and Gould, 1999). The observation that residual ventral VD synapses are retained with RNAi disruption of arx-5 function in an unc-55 mutant (Fig. 3F, G) suggests that
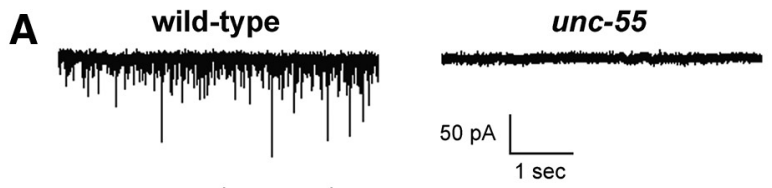

unc-55; irx-1(csRNAi)
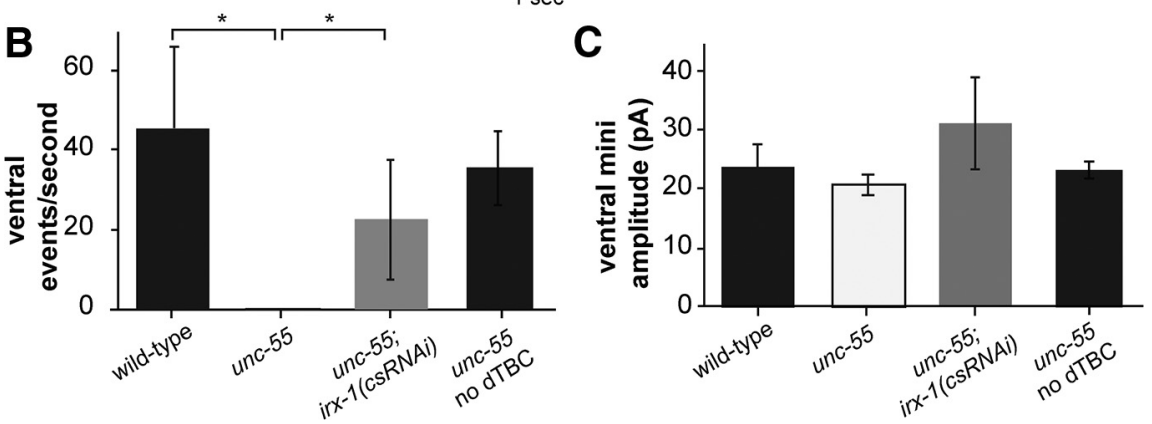

Figure 7. irx- 1 knockdown restores ventral GABAergic synaptic output in unc- 55 mutants. $A$, mPSCs are abundant in ventral body muscles of wild-type and unc-55; irx-1(csRNAi) animals but are rare in unc- 55 mutants. The animals in $\boldsymbol{A}$ were treated with dTBC to block cholinergic activity. $\boldsymbol{B}$, Quantification of results in $\boldsymbol{A}$ shows restoration of ventral mPSCs to unc -55 mutants treated with irx-1(csRNAi). As a control, unc- 55 animals were recorded without dTBC. Robust cholinergic mPSCs in unc-55 animals in the absence of dTBC indicate that the Unc- 55 defect is specific to GABAergic synapses. ${ }^{*} p<0.05$, Mann-Whitney Utest. $N=4-6$ for each genotype. Error bars indicate SD. C, Average mPSC amplitude is similar for all recordings, indicating that mPSCs are quantal. $N=4-6$ for each genotype. Error bars indicate SD.
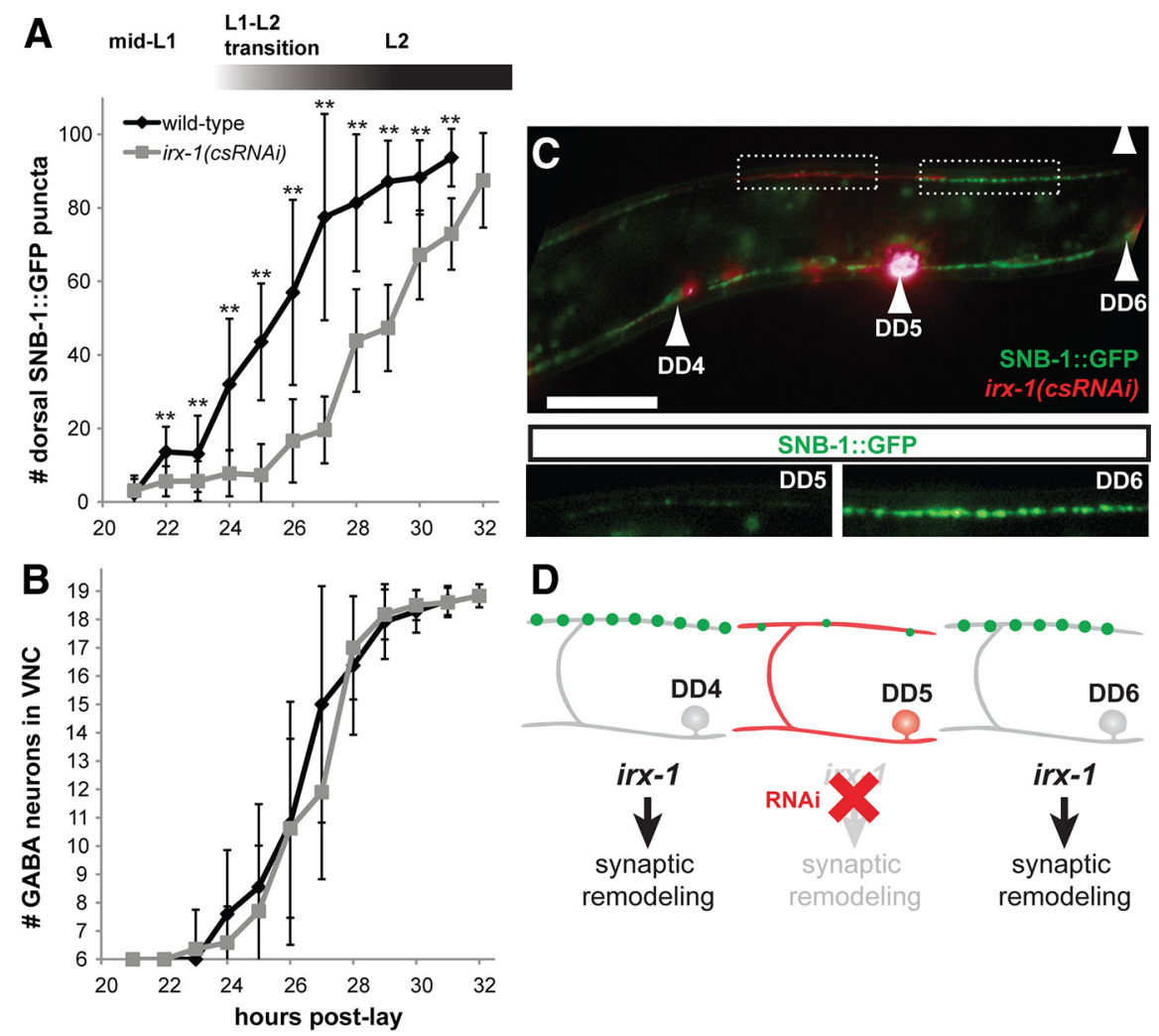

Figure 8. $\quad$ irx-1 function is required for the normal progression of synaptic remodeling in DD motor neurons. $A, B, D D$ synaptic remodeling is delayed in irx-1(cSRNAi) animals. $A$, DD remodeling was quantified by counting dorsal SNB-1::GFP puncta during L1-L2 larval stages. In wild-type (SNB-1::GFP;GABA::mCherry-expressing) larvae, DD remodeling begins at 22-24 $\mathrm{h}$ (see Materials and Methods) in L1 larvae and is completed by the L2 stage. In contrast, remodeling is delayed in irx-1(csRNAi) larvae. ${ }^{* *} p<0.01$, wild-type versus irx-1(csRNAi), Student's $t$ test. Error bars indicate SD. $N=6-21$ for each genotype at each hour; results pooled from three independent experiments. $\boldsymbol{B}$, Generation of GABAergic motor neurons is not disrupted by abrogation of irx-1 activity. The normal complement of six embryonic DD motor neurons was observed in both wild-type and irx-1(csRNAi) larvae before remodeling $(t=21 \mathrm{~h})$. The rate of appearance of the 13 postembryonic VD motor neurons was not delayed in irx-1(csRNAi) larvae compared with wild type. $\mathbf{C}, \mathbf{D}$, irx-1(csRNAi) causes cell-autonomous delays in DD motor neuron synaptic remodeling. Image of a mosaic irx-1(csRNAi)-expressing larva at $29 \mathrm{~h}$ when DD remodeling is largely complete in wild type (top panel). Strong expression of the irx-1(csRNAi) transgene, indicated by the mCherry marker, in DD5 but not DD6 (or DD4) is correlated with reduced dorsal SNB-1::GFP puncta in DD5 compared with DD6 (inset panels). 

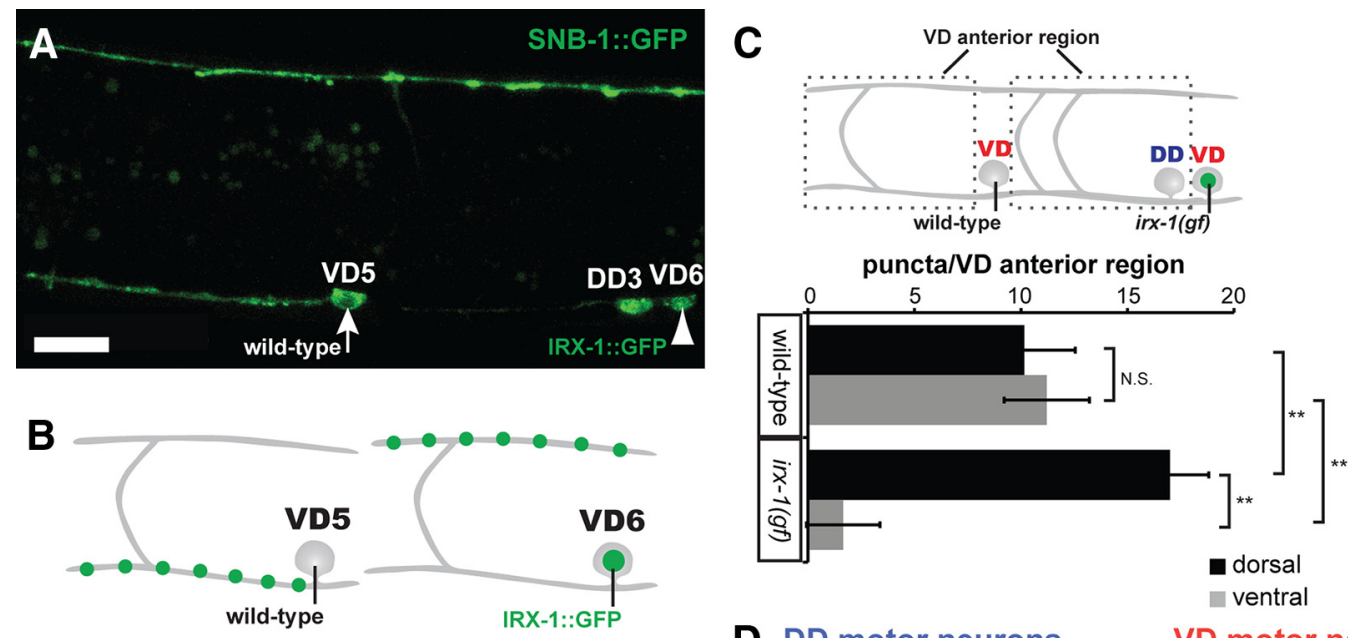

unc-55

unc-55
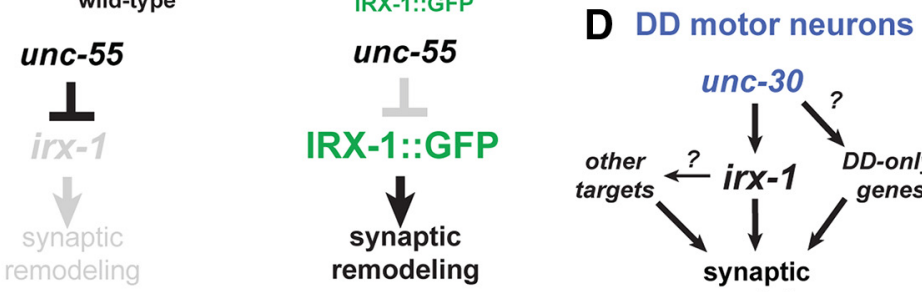

VD motor neurons
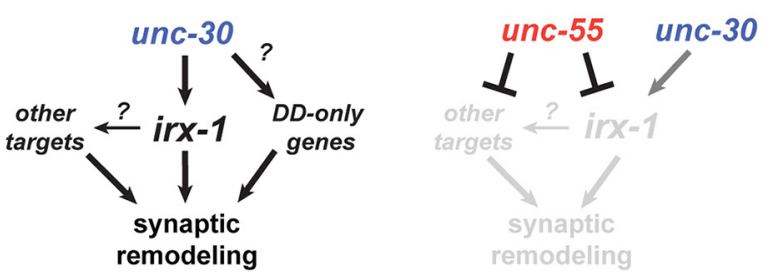

Figure 9. Ectopic irx-1 expression is sufficient to drive remodeling in VD motor neurons. A, Mosaic expression of GFP-labeled IRX-1 (IRX-1::GFP) with the ttr-39 promoter labels the VD6 nucleus (arrowhead) but not VD5. Residual SNB-1::GFP in the VD5 cell soma is excluded from the nucleus (arrow) (adult stage). Note absence of ventral SNB-1::GFP puncta in the ventral process of VD6 and corresponding enhanced SNB-1::GFP expression in the dorsal VD6 process. $\boldsymbol{B}$, Schematic diagram of results from $\boldsymbol{A}$. In VD6, unc-55 represses endogenous irx-1 but does not block IRX-1::GFP expression from the ttr-39 promoter, which drives remodeling. C, Quantification of the irx-1(gf) phenotype. SNB-1::GFP puncta were counted in the dorsal and ventral nerve cords within each VD anterior region for wild-type versus IRX-1::GFP-expressing VD neurons as indicated. ${ }^{* *} p<0.01$, two-way ANOVA with Bonferroni's multiple-comparison test; N.S., not significant. D, Genetic pathways that control synaptic remodeling. unc -55 prevents expression of irx- 1 and 48 additional factors (18 conserved) to block synaptic remodeling in VD motor neurons. unc-30 promotes irx-1 expression. irx-1 is normally expressed in DD motor neurons where it drives synaptic remodeling in parallel to at least one additional partially redundant pathway.

reorganization of the actin cytoskeleton is required for their removal. This finding is intriguing because F-actin (filamentous actin) is a known synaptic component and has been shown to promote synaptic assembly and function (Dillon and Goda, 2005). Here, our results are consistent with an alternative model in which actin polymerization leads to disassembly of the presynaptic machinery. This cytoskeletal reorganization could also depend on the unc-55-regulated synaptic remodeling gene, cnt-1, which encodes a conserved Centaurin- $\beta$ ArfGAP (Arf GTPaseactivating protein). Arf family GTPases are key regulators of the actin cytoskeleton and vesicular trafficking in neurons (Myers and Casanova, 2008; Klassen et al., 2010). Our results suggest that that activation of a specific Arf GTPase by CNT-1 is required for synaptic remodeling in GABAergic motor neurons.

Other downstream effectors of synaptic remodeling revealed by our approach could fulfill additional roles. For example, RNAi knockdown of the DEG/ENaC cation channel subunit protein UNC-8 impedes the removal of ventral GABAergic motor neuron synaptic components in unc-55 mutants. This finding indicates that wild-type UNC-8 protein functions in a biological process that destabilizes ventral synapses in remodeling VD motor neurons. A potential role for UNC-8 in synaptic plasticity is consistent with findings that members of the closely related acidsensing ion channel (ASIC) family are synaptically localized and contribute to learning and memory mechanisms that depend on neuronal activity (Coryell et al., 2007; Voglis and Tavernarakis, 2008; Zha et al., 2009). Our evidence suggests the additional possibility that ASIC proteins are also involved in developmentally regulated synaptic plasticity. In this context, we suggest that
UNC-8 may fulfill an indirect role in the removal of ventral GABAergic synapses by stabilizing dorsal outputs.

A notable group of UNC-55-regulated genes revealed by our study points to an important role for the extracellular matrix (ECM) in synaptic remodeling. The mammalian neuromuscular junction is bisected by a basal lamina that courses between presynaptic and postsynaptic membranes (Chiu and Sanes, 1984). This characteristic feature is preserved in C. elegans (White et al., 1986), and thus it is reasonable to expect that remodeling of GABAergic synapses could involve specific changes in ECM architecture. One of the ECM-related genes in our list, clec-44, encodes a presumptive $\mathrm{C}$-lectin cell surface protein. A likely role for clec-44 in synaptogenesis is supported by the finding that a related C-lectin, clec-38, contributes to the assembly of the presynaptic apparatus in C. elegans GABAergic motor neurons (Kulkarni et al., 2008). Potential ECM components regulated by unc-55 include three collagen genes, col-12, col-54, and col-77. Collagens fulfill multiple roles at the neuromuscular synapse such as anchoring neurotransmitter receptors and promoting synaptic maturation and maintenance (Fox, 2008; Latvanlehto et al., 2010). Recent reports indicate that GABAergic neurons in the mouse hippocampus (Su et al., 2010) and in the C. elegans motor circuit (Ackley et al., 2003) secrete specific collagens that promote synaptogenesis. Finally, our study found that two of the conserved synaptic remodeling genes encode the predicted matrix metalloproteases (MMPs), nas-38 (a nematode astacin homologous to mammalian BMP1) and T19D2.1 [predicted to function as ADAM (a disintegrin and metalloproteinase)]. Important roles for MMPs in synaptic plasticity are indicated by the findings 
that injury-induced remodeling and activity-dependent LTP in the hippocampus require MMP activity (Ethell and Ethell, 2007; Wang et al., 2008).

The phenomenon of synaptic plasticity is widely observed and crucially important to the development and maintenance of functional nervous systems. Our approach has now discovered a large and diverse array of protein types with necessary roles in synaptic remodeling. Future studies that explore the specific functions of these components offer a powerful opportunity to elucidate the molecular mechanism of this fundamental biological process.

\section{References}

Ackley BD, Kang SH, Crew JR, Suh C, Jin Y, Kramer JM (2003) The basement membrane components nidogen and type XVIII collagen regulate organization of neuromuscular junctions in Caenorhabditis elegans. J Neurosci 23:3577-3587.

Armentano M, Chou SJ, Tomassy GS, Leingärtner A, O'Leary DD, Studer M (2007) COUP-TFI regulates the balance of cortical patterning between frontal/motor and sensory areas. Nat Neurosci 10:1277-1286.

Bamber BA, Beg AA, Twyman RE, Jorgensen EM (1999) The Caenorhabditis elegans unc-49 locus encodes multiple subunits of a heteromultimeric GABA receptor. J Neurosci 19:5348-5359.

Barnes SJ, Finnerty GT (2010) Sensory experience and cortical rewiring. Neuroscientist 16:186-198.

Boulanger A, Clouet-Redt C, Farge M, Flandre A, Guignard T, Fernando C, Juge F, Dura JM (2011) ftz-f1 and Hr39 opposing roles on EcR expression during Drosophila mushroom body neuron remodeling. Nat Neurosci $14: 37-44$.

Brenner S (1974) The genetics of Caenorhabditis elegans. Genetics 77:71-94.

Bürglin TR (1997) Analysis of TALE superclass homeobox genes (MEIS, PBC, KNOX, Iroquois, TGIF) reveals a novel domain conserved between plants and animals. Nucleic Acids Res 25:4173-4180.

Cavodeassi F, Modolell J, Gómez-Skarmeta JL (2001) The Iroquois family of genes: from body building to neural patterning. Development 128:2847-2855.

Cheng CW, Chow RL, Lebel M, Sakuma R, Cheung HO, Thanabalasingham V, Zhang X, Bruneau BG, Birch DG, Hui CC, McInnes RR, Cheng SH (2005) The Iroquois homeobox gene, Irx5, is required for retinal cone bipolar cell development. Dev Biol 287:48-60.

Chiu AY, Sanes JR (1984) Development of basal lamina in synaptic and extrasynaptic portions of embryonic rat muscle. Dev Biol 103:456-467.

Cinar H, Keles S, Jin Y (2005) Expression profiling of GABAergic motor neurons in Caenorhabditis elegans. Curr Biol 15:340-346.

Coryell MW, Ziemann AE, Westmoreland PJ, Haenfler JM, Kurjakovic Z, Zha XM, Price M, Schnizler MK, Wemmie JA (2007) Targeting ASIC1a reduces innate fear and alters neuronal activity in the fear circuit. Biol Psychiatry 62:1140-1148

Dillon C, Goda Y (2005) The actin cytoskeleton: integrating form and function at the synapse. Annu Rev Neurosci 28:25-55.

Ding M, Chao D, Wang G, Shen K (2007) Spatial regulation of an E3 ubiquitin ligase directs selective synapse elimination. Science 317:947-951.

Earls LR, Hacker ML, Watson JD, Miller DM 3rd (2010) Coenzyme Q protects Caenorhabditis elegans GABA neurons from calcium-dependent degeneration. Proc Natl Acad Sci U S A 107:14460-14465.

Ethell IM, Ethell DW (2007) Matrix metalloproteinases in brain development and remodeling: synaptic functions and targets. J Neurosci Res 85:2813-2823.

Feldman DE (2009) Synaptic mechanisms for plasticity in neocortex. Annu Rev Neurosci 32:33-55.

Finney M, Ruvkun G (1990) The unc-86 gene product couples cell lineage and cell identity in C. elegans. Cell 63:895-905.

Flavell SW, Kim TK, Gray JM, Harmin DA, Hemberg M, Hong EJ, Markenscoff-Papadimitriou E, Bear DM, Greenberg ME (2008) Genome-wide analysis of MEF2 transcriptional program reveals synaptic target genes and neuronal activity-dependent polyadenylation site selection. Neuron 60:1022-1038.

Fox MA (2008) Novel roles for collagens in wiring the vertebrate nervous system. Curr Opin Cell Biol 20:508-513.

Gally C, Bessereau JL (2003) GABA is dispensable for the formation of junc- tional GABA receptor clusters in Caenorhabditis elegans. J Neurosci 23:2591-2599.

Gómez-Skarmeta JL, Modolell J (2002) Iroquois genes: genomic organization and function in vertebrate neural development. Curr Opin Genet Dev 12:403-408.

Hallam SJ, Jin Y (1998) lin-14 regulates the timing of synaptic remodelling in Caenorhabditis elegans. Nature 395:78-82.

Hammock EA, Eagleson KL, Barlow S, Earls LR, Miller DM 3rd, Levitt P (2010) Homologs of genes expressed in Caenorhabditis elegans GABAergic neurons are also found in the developing mouse forebrain. Neural Dev 5:32.

Hashimoto K, Ichikawa R, Kitamura K, Watanabe M, Kano M (2009) Translocation of a "winner" climbing fiber to the Purkinje cell dendrite and subsequent elimination of "losers" from the soma in developing cerebellum. Neuron 63:106-118.

Irizarry RA, Bolstad BM, Collin F, Cope LM, Hobbs B, Speed TP (2003) Summaries of Affymetrix GeneChip probe level data. Nucleic Acids Res 31:e15.

Kamath RS, Fraser AG, Dong Y, Poulin G, Durbin R, Gotta M, Kanapin A, Le Bot N, Moreno S, Sohrmann M, Welchman DP, Zipperlen P, Ahringer J (2003) Systematic functional analysis of the Caenorhabditis elegans genome using RNAi. Nature 421:231-237.

Kennedy S, Wang D, Ruvkun G (2004) A conserved siRNA-degrading RNase negatively regulates RNA interference in C. elegans. Nature 427:645-649.

Kim BJ, Takamoto N, Yan J, Tsai SY, Tsai MJ (2009) Chicken ovalbumin upstream promoter-transcription factor II (COUP-TFII) regulates growth and patterning of the postnatal mouse cerebellum. Dev Biol 326:378-391.

Klassen MP, Wu YE, Maeder CI, Nakae I, Cueva JG, Lehrman EK, Tada M, Gengyo-Ando K, Wang GJ, Goodman M, Mitani S, Kontani K, Katada T, Shen K (2010) An Arf-like small G protein, ARL-8, promotes the axonal transport of presynaptic cargoes by suppressing vesicle aggregation. Neuron 66:710-723.

Kulkarni G, Li H, Wadsworth WG (2008) CLEC-38, a transmembrane protein with C-type lectin-like domains, negatively regulates UNC-40mediated axon outgrowth and promotes presynaptic development in Caenorhabditis elegans. J Neurosci 28:4541-4550.

Latvanlehto A, Fox MA, Sormunen R, Tu H, Oikarainen T, Koski A, Naumenko N, Shakirzyanova A, Kallio M, Ilves M, Giniatullin R, Sanes JR, Pihlajaniemi T (2010) Muscle-derived collagen XIII regulates maturation of the skeletal neuromuscular junction. J Neurosci 30:12230-12241.

Lodato S, Tomassy GS, De Leonibus E, Uzcategui YG, Andolfi G, Armentano M, Touzot A, Gaztelu JM, Arlotta P, Menendez de la Prida L, Studer M (2011) Loss of COUP-TFI alters the balance between caudal ganglionic eminence- and medial ganglionic eminence-derived cortical interneurons and results in resistance to epilepsy. J Neurosci 31:4650-4662.

Machesky LM, Gould KL (1999) The Arp2/3 complex: a multifunctional actin organizer. Curr Opin Cell Biol 11:117-121.

Maduro M, Pilgrim D (1995) Identification and cloning of unc-119, a gene expressed in the Caenorhabditis elegans nervous system. Genetics 141:977-988.

Matsumoto K, Nishihara S, Kamimura M, Shiraishi T, Otoguro T, Uehara M, Maeda Y, Ogura K, Lumsden A, Ogura T (2004) The prepattern transcription factor Irx2, a target of the FGF8/MAP kinase cascade, is involved in cerebellum formation. Nat Neurosci 7:605-612.

Mlodzik M, Hiromi Y, Weber U, Goodman CS, Rubin GM (1990) The Drosophila seven-up gene, a member of the steroid receptor gene superfamily, controls photoreceptor cell fates. Cell 60:211-224.

Mukherjee K, Bürglin TR (2007) Comprehensive analysis of animal TALE homeobox genes: new conserved motifs and cases of accelerated evolution. J Mol Evol 65:137-153.

Myers KR, Casanova JE (2008) Regulation of actin cytoskeleton dynamics by Arf-family GTPases. Trends Cell Biol 18:184-192.

Park M, Watanabe S, Poon VY, Ou CY, Jorgensen EM, Shen K (2011) CYY$1 /$ Cyclin $\mathrm{Y}$ and CDK-5 differentially regulate synapse elimination and formation for rewiring neural circuits. Neuron 70:742-757.

Poon VY, Klassen MP, Shen K (2008) UNC-6/netrin and its receptor UNC-5 locally exclude presynaptic components from dendrites. Nature 455:669-673.

Richmond JE, Jorgensen EM (1999) One GABA and two acetylcholine re- 
ceptors function at the C. elegans neuromuscular junction. Nat Neurosci 2:791-797.

Roy PJ, Stuart JM, Lund J, Kim SK (2002) Chromosomal clustering of muscle-expressed genes in Caenorhabditis elegans. Nature 418:975-979.

Sarov M, Schneider S, Pozniakovski A, Roguev A, Ernst S, Zhang Y, Hyman AA, Stewart AF (2006) A recombineering pipeline for functional genomics applied to Caenorhabditis elegans. Nat Methods 3:839-844.

Schuske K, Beg AA, Jorgensen EM (2004) The GABA nervous system in C. elegans. Trends Neurosci 27:407-414.

Shan G, Kim K, Li C, Walthall WW (2005) Convergent genetic programs regulate similarities and differences between related motor neuron classes in Caenorhabditis elegans. Dev Biol 280:494-503.

Spencer WC, Zeller G, Watson JD, Henz SR, Watkins KL, McWhirter RD, Petersen S, Sreedharan VT, Widmer C, Jo J, Reinke V, Petrella L, Strome S, Von Stetina SE, Katz M, Shaham S, Rätsch G, Miller DM 3rd (2011) A spatial and temporal map of C. elegans gene expression. Genome Res 21:325-341.

Su J, Gorse K, Ramirez F, Fox MA (2010) Collagen XIX is expressed by interneurons and contributes to the formation of hippocampal synapses. J Comp Neurol 518:229-253.

Tang K, Xie X, Park JI, Jamrich M, Tsai S, Tsai MJ (2010) COUP-TFs regulate eye development by controlling factors essential for optic vesicle morphogenesis. Development 137:725-734.

Tissot M, Stocker RF (2000) Metamorphosis in Drosophila and other insects: the fate of neurons throughout the stages. Prog Neurobiol 62:89-111.

Tomassy GS, De Leonibus E, Jabaudon D, Lodato S, Alfano C, Mele A, Macklis JD, Studer M (2010) Area-specific temporal control of corticospinal motor neuron differentiation by COUP-TFI. Proc Natl Acad Sci U S A 107:3576-3581.

Voglis G, Tavernarakis N (2008) A synaptic DEG/ENaC ion channel mediates learning in C. elegans by facilitating dopamine signalling. EMBO J 27:3288-3299.

Von Stetina SE, Fox RM, Watkins KL, Starich TA, Shaw JE, Miller DM 3rd (2007a) UNC-4 represses CEH-12/HB9 to specify synaptic inputs to VA motor neurons in C. elegans. Genes Dev 21:332-346.

Von Stetina SE, Watson JD, Fox RM, Olszewski KL, Spencer WC, Roy PJ, Miller DM 3rd (2007b) Cell-specific microarray profiling experiments reveal a comprehensive picture of gene expression in the C. elegans nervous system. Genome Biol 8:R135.

Walthall WW (1990) Metamorphic-like changes in the nervous system of the nematode Caenorhabditis elegans. J Neurobiol 21:1085-1091.

Walthall WW, Plunkett JA (1995) Genetic transformation of the synaptic pattern of a motoneuron class in Caenorhabditis elegans. J Neurosci 15:1035-1043.

Wang XB, Bozdagi O, Nikitczuk JS, Zhai ZW, Zhou Q, Huntley GW (2008) Extracellular proteolysis by matrix metalloproteinase-9 drives dendritic spine enlargement and long-term potentiation coordinately. Proc Natl Acad Sci U S A 105:19520-19525.

Warming S, Costantino N, Court DL, Jenkins NA, Copeland NG (2005) Simple and highly efficient BAC recombineering using galK selection. Nucleic Acids Res 33:e36.

White JG, Southgate E, Thomson JN, Brenner S (1976) The structure of the ventral nerve cord of Caenorhabditis elegans. Philos Trans R Soc Lond B Biol Sci 275:327-348.

White JG, Albertson DG, Anness MA (1978) Connectivity changes in a class of motoneurone during the development of a nematode. Nature 271:764-766.

White JG, Southgate E, Thomson JN, Brenner S (1986) The structure of the nervous system of the nematode Caenorhabditis elegans. Philos Trans R Soc Lond B Biol Sci 314:1-340.

Yeh E, Kawano T, Weimer RM, Bessereau JL, Zhen M (2005) Identification of genes involved in synaptogenesis using a fluorescent active zone marker in Caenorhabditis elegans. J Neurosci 25:3833-3841.

Zha XM, Costa V, Harding AM, Reznikov L, Benson CJ, Welsh MJ (2009) ASIC2 subunits target acid-sensing ion channels to the synapse via an association with PSD-95. J Neurosci 29:8438-8446.

Zhong M, Niu W, Lu ZJ, Sarov M, Murray JI, Janette J, Raha D, Sheaffer KL, Lam HY, Preston E, Slightham C, Hillier LW, Brock T, Agarwal A, Auerbach R, Hyman AA, Gerstein M, Mango SE, Kim SK, Waterston RH, et al. (2010) Genome-wide identification of binding sites defines distinct functions for Caenorhabditis elegans PHA-4/FOXA in development and environmental response. PLoS Genet 6:e1000848.

Zhou HM, Walthall WW (1998) UNC-55, an orphan nuclear hormone receptor, orchestrates synaptic specificity among two classes of motor neurons in Caenorhabditis elegans. J Neurosci 18:10438-10444. 